\title{
Testing the Effect of Capital Flow on Stock Market Return: Empirical Evidence from A Study of United States of America
}

\author{
LAI, Ping-fu (Brian) \\ Associate Professor \\ College of Global Talents \\ Beijing Institute of Technology, Zhuhai, China \\ NGOK Chun-kit (Patrick) \\ ABRS International Consultancy
}

\begin{abstract}
This research is to analyze the effect of capital flow's path on the return of the stock market in the United States. First, eight variables are tested using Pearson Correlations to explore the relationships between stock market return and Standard Multiple Regression is conducted to investigate the prediction ability. Second, Pearson Correlations are repeated to find out whether the eight variables and stock market return have lead-lag relationships and run a regression again. Third, the data set is divided into two panels representing different investment environments. Panel A is when the growth rate of M1 greater than the growth rate of M2 while Panel B is when the growth rate of M2 greater than the growth rate of M1. The findings of this research show that six out of eight variables have lead-lag relationships with the stock market return. When dividing into two panels, most of the eight variables have a more slightly correlated relation with the stock market return and the prediction ability of the variables is stronger than the original data set.
\end{abstract}

Keywords: Capital flow, Lead-lag relation, Stock market return

\section{Introduction}

The stock is a fundraising tool for listed companies and is also an investment instrument for investors. Investing in stocks is often to have expectations on the economic outlook or the individual company's outlook. Apart from this, it is well known that the change of the stock market and capital flows are closely correlated. When a great amount of capital (sometimes hot money) rushes into the stock market, the upward movements of share prices are often driven due to actively buying. Oppositely, when capital leaves the market, share prices often fall due to actively selling. The judgment process of capital flows is relatively complex and not easy to grasp. However, it has an important role to analyze the trend of the stock market. (Gerig 2007)

Not surprisingly, the worldwide stock markets were also benefited from the quantitative easing policies. For example, the US Federal Reserve implemented three rounds of quantitative easing since 2008 while the Dow rose from the lowest level 6626 to around 15000 points until now. (Culver 2010) Experienced in a wave after wave of falling price, investors are increasingly pessimistic about the stock market and withdraw their investments. Thus, whether from the perspective of enterprises or investors, when M2 outstrips significantly M1 which is in a low-cost investment environment, sufficient capital will slowly push forward the economy and this could be a signal that the bear market is bottomed out. (Alshogeathri 2011).Oppositely, when capital outflows from the market which lowers the share prices, the exchange rate often depreciates. (Tas 2008) The Federal Reserve uses open market operations such as buying and selling government bonds in the market to influence the supply of money so as to encourage or discourage the lending from the bank. (Yin 2007) The sufficiency of short-term capital would generally have an impact on the stock market. Basically, there is a positive relationship between them.

Like the international financial crisis in 2008 , the stock market crashed while the bond prices appreciated a lot in this year. Intuitively, the relationship between the two markets described as "seesaw", that is, they have an inverse relationship because of the asset allocation effect. It was, therefore, conclude this experience: if you buy stocks or equity mutual funds when the market is bull and if you buy bonds or bond mutual funds when the market is bear, then whether it is bull or bear market, you can earn money as well. (Li 2007) The reason is that when the economy is expanding or interest rate is at a low level, bondholders tend to withdraw their investment from the bond market to other assets for earning a higher profit. 
At this time, the return of stock market is usually relatively high which makes the few percents of fixed interest becoming dwarf. Some mutual funds will enhance the highest possible stake limits and sometimes even consider borrow funds through the repo market for purchasing new shares and participating non-public issuance and so on. (Oh and Parwada 2007) Therefore, capital will flow from the bond market to the stock market. Bond prices will perform weaker. In turn, when the stock market is bear or interest rate is at a high level, investors will reduce the equity positions of which the withdrawn funds will tend to come to bank deposit and bond markets. Simultaneously, mutual funds will push their proportion of bond holdings to the highest limit. Because of the expansion of demand, the bond price will appreciate. Capital will flow from the stock market to bond market. However, this logic is assumed that the amount of funds in the market does not change. Stock and bond markets may have a more complex relationship if the total funds increase or decrease. Capital may inflow to or outflow from the bond and stock markets simultaneously which both bond and stock prices will appreciate or depreciate. In fact, there did not find a consistent conclusion referring to the literature in the past. (Li 2007). When the economy expands, people have more capital and more willing to buy property whatever for investment or private use. Property prices will appreciate because of the higher demand. If the economy shrinks, people may tend to sell the property for cash or unwilling to buy. Property price will depreciate because of lower demand. (Beracha 2007)

Another investment tool which usually considered as a hedge is gold. (Capie et. al. 2005) The gold standard used to be a monetary policy in many countries before. In the past, gold started to act as a medium of exchange because of its rarity. With the widespread monetary function for trading, it became the most suitable choice for the world's currency. Various countries' currency system linked to it and therefore the gold standard was established. However, most countries with huge war debts left the gold standard after World War I. Since then gold was widely supplanted by fiat currency and finally collapsed. Nowadays, gold continues to be regarded as a "quasi-currency" which is accepted internationally. Similar to the foreign exchange reserves and government bonds, gold reserves have an important position in the fiscal reserves of various countries. Not only it protects the currency of one's country but also hedges losses caused by the depreciation of US dollar. It still has a wide range of application and regards as a way of storing wealth. For investors, gold is regarded as a hedge against inflation or other economic disruption. From the past, it has appeared that when the economy shrinks, the stock market decline while gold price will appreciate. (Coudert and Raymond 2012)

Apart from the above, another investment market is the commodity market. Stevenson (2004) suggested that the commodity and stock market have an inverse relation. The rising and falling time were not completely accurate, but an inverse trend could be seen. The reason is that when the purchasing power of money declines, commodity prices will rise. At this time, the operating costs of enterprises increase and thereby lower the enterprises' profitability which likely causes the shrink of share prices. On the other hand, if the purchasing power of money rises up, commodity prices will fall. The operating cost of enterprises reduces this time and profitability will be increased which likely favor the rise of share prices.

\section{Literature Review}

The flowing of capital in investment market plays an important role in analyzing the trend of the stock market. Capital can flow from one country to another, between money supply M1 and M2, in and out of the banking system and from one asset to another. Hashemzadeh and Taylor (1988) using the weekly data to investigate the relationship between S\&P 500, the return of US Treasury bills and money supply (M1). The results of Granger-Sims's causality tests showed that there was a feedback relationship between the S\&P 500 and M1 but the relationship between US Treasury bill and S\&P 500 was not conclusive. Malliaris and Urrutia (1991) studied the causal relationship between S\&P 500, M1 and Industrial production. They collected monthly data from Jan 1970 to June 1989 for their study. The result of the Granger causality test indicated that M1 led the S\&P 500. Darrat and Dickens (1999) used the same data set as Malliaris and Urrutia (1991). They further found that the three variables had causal interrelationship and were integrated. The results implicated that stock market lead economic activity and monetary policy. Abdullah and Hayworth (1993) examined the relationships between seven variables and the fluctuations of the US stock market's monthly return using impulse response analysis, vector Autoregressions and Granger causality tests. One of the results indicated that M1 was positively correlated with S\&P 500. The similar result was also examined by Dhakai, Kandil, and Sharma (1993). They investigated the linkage of five US macroeconomic variables in the stock market over the period 1973 to 1991 . The VAR results showed that the stock market return was significantly impacted by the change of money supply. Thornton (1993) investigated the lead-lag linkage between the UK stock market and GDP, money supply M0 and M5. He used quarterly data from 1963 to 1990. The results suggested that stock prices in FTSE100 tend to lead M5. 
Besides, M0 and M5 volatility and stock price volatility had feedback effects. Ibrahim (1999) observed data from the period January 1977 to June 1996 to test the dynamic relationships between monthly Malaysian stock prices and seven macroeconomic variables such as M1, M2, domestic credit, and exchange rate. The cointegration and Granger tests results indicated that domestic credits were inefficient with stock prices. M2 were cointegrated with stock prices while M1 did not have a long-run relationship with stock prices.

For the study of the exchange rate, Ratanapakorn and Sharma (2007) studied the long and short run relationships between six variables and the S\&P 500. The research collected monthly data from January 1975 to April 1999. The findings showed that the money supply and exchange rate were positively correlated with the stock prices. Wenshwo (2002) used a GARCH model to access whether the stock returns and volatilities were affected by the depreciation of the currency in Hong Kong, Thailand, Taiwan, South Korea and Singapore. The study evidenced that exchange rate depreciation influenced stock return during the Asian crisis (1997-1999). However, the study of Patra and Poshakwale (2006) had a different result. They investigated whether there had long and short-run relationships between the monthly Greek stock price index and several macroeconomic indicators such as the exchange rate and money supply. They collected data from 1990 to 1999. The results of vector error correction models, Johansen cointegration tests, and Granger causality tests indicated that only the exchange rate did not have the relationship with stock prices.

For the literature about bank credit, Kim and Moreno (1994) studied the effect of bank lending on Japan's stock prices from January 1970 to May 1993. They found that bank credit and stock prices had a positive correlation in two subperiods. Also, the volatility of bank credit influenced significantly to the volatility of Japan's stock prices. Ibrahim (2006) explored the relationship between quarterly Malaysia stock prices with bank loans. The data were collected from January 1978 to February 1998 and were analyzed by VAR tests. The results showed that bank loans were positively influenced by stock prices but stock prices were not influenced by stock prices.

The relationship between bond and stock is a hot topic for researchers. Their relationship is important for asset allocation. Downing et al. (2006) used a VAR model to explore the contemporaneous correlation between bond and stock returns but could not find any lead-lag relation between them. Another research using weekly data was studied by $\mathrm{Li}$ (2007). The research used the ADCC model to examine the time-varying correlations between bond and stock returns. The results provided evidence that the stock had an average negative correlation with bond but it was weak. Their correlation was affected by some key economic factors such as oil price shock and the yield spread. Two phenomena "flight to quality" and "flight from quality" were discovered by Hartmann et al. (2001). "Flight to quality" is that investors would move their investments from stocks to bonds when the stock market is bear. In contrast, "flight from quality" is that investors would move their investments back to stocks from bonds when the stock market is bull. Baur and Lucey (2006), Connolly et al. (2005) and Gulko (2002) agreed the phenomena that the two flights affect the correlation of stock and bond.

Beracha (2007) investigated the relationship between stock market return and the performance of home price. The findings implicated that the price and trading volume of stocks were affected by the change in home prices. The return of stocks and bonds were positively correlated with the change of real-estates' prices. Sutton (2002) examined the effect of consumer's spending and investing behaviors on house prices. The research analyzed quarterly data from 1970 to 2002 in six countries by the VAR model. The findings indicated that the volatility of house price was caused by the volatility of national income, interest rate and stock prices.

Milunovich et al. (2011) explored the linkage between the Australian stock market and international commodity prices. They used constructed returns and found that the commodity prices had a significant effect on the stock market. Hondroyiannis and Papapetrou (2001) examined the dynamic relationship between the return of Greek stock market and macroeconomic variables such as exchange rate and real oil price by a multivariate vector autoregressive VAR model. The data were collected monthly from January 1984 to September 1999. They found that the stock price changes can be explained by the price of oil. Lombardi and Ravazzolo (2013) accessed whether the correlation between commodity prices and stock prices at an increase. Various correlation tests were conducted. They suggested that the higher correlation had implication for portfolio setting. Commodity could act as a hedge.

Li (2013) studied the linkage between gold, US dollar, and stock market return. The study used data from 2001 to 2012 and was analyzed by ADCC-CARCH model. The findings showed that the gold price was negatively correlated with stock returns during the financial tsunami and European sovereign debt crisis. However, the overall correlation between them was positive. Another study examined whether gold was a hedge against stocks in emerging and developed countries. Baur and Mcdermott (2010) gathered 30 years of data from 1979 to 2009. The results provided evidence that gold acted as a safe haven for US and European stocks but not for Japan, Canada, Australia, and BRIC. Hillier et al (2006) analyzed the daily data from 1976 to 2004 for three metals including gold. Results suggested that gold had a low correlation with the return of stocks. It had some diversification benefits for portfolio settings. 


\section{Data Collection}

This research uses data which are the historical closed prices or values released on a particular day of each week except that gold price uses the fixing price 10:30 AM (London time). I use a weekly frequency time-series data set from Jan 2005 to June 2013, a time period that the investment market experienced up and down, to explore the relationship between stock market and capital flow in the investment market. Within the time, the investment market moved especially dramatic when the expansion of the financial tsunami in 2008 and the implementation of quantitative easing policies by major countries' central bank. I use the return of Standard and Poor's 500 index (S\&P500) as a proxy for the US stock market's return. It is a US stock market index based on the market capitalizations of 500 large companies having common stocks listed on the New York Stock Exchange (NYSE) or National Association Securities Dealers Automated Quotations (NASDAQ).

Capital except for flows between investment markets, it also flows between different assets. Bond is another commonly used investment instruments among them. The return of Vanguard Total Bond Market Index Fund is used as a proxy for the US bond return. The investment seeks the performance of a broad, market-weighted bond index. The fund employs an indexing investment approach designed to track the performance of the Barclays U.S. Aggregate Float Adjusted Index. This Index represents a wide spectrum of public, investment-grade, taxable, fixed income securities in the United States-including government, corporate, and international dollar-denominated bonds, as well as mortgagebacked and asset-backed securities-all with maturities of more than 1 year. The Fund invests by sampling the index, meaning that is held a broadly diversified collection of securities that, in the aggregate, approximates the full index in terms of key risk factors and other characteristics. All of the Fund's investments will be selected through the sampling process, and at least $80 \%$ of the fund's assets will be invested in bonds held in the index. The Fund maintains a dollarweighted average maturity consistent with that of the index, which generally ranges between 5 and 10 years. For the return of property, the prices of Inland Real Estate Corporation are used. Inland Real Estate Corporation currently is a Real Estate Investment Trust ("REIT") based in Oak Brook, Illinois. It acquires, owns and manages neighborhood and community retail centers located primarily in the United States. It owns interests in and manages 161 properties, with an approximate 15 million square feet of real estate totaling nearly $\$ 2$ billion in asset acquisition value. With regard to the return of commodity, the prices of Thomson Reuters / Jefferies CRB Index are used. It is the most widely recognized measure of global commodities markets. As a benchmark, the Index is designed to provide timely and accurate representation of a long-only, broadly diversified investment in commodities through a transparent and disciplined calculation methodology. It currently is made up of 19 commodities as quoted on the NYMEX, CBOT, LME, CME and COMEX exchanges. These are sorted into 4 groups, each with different weightings. These groups are:

-Petroleum-based products (based on their importance to global trade, always makeup 33\% of the weightings)

- Liquid assets

-Highly liquid assets

-Diverse commodities.

The index comprises 19 commodities: Aluminum, Cocoa, Coffee, Copper, Corn, Cotton, Crude Oil, Gold, Heating Oil, Lean Hogs, Live Cattle, Natural Gas, Nickel, Orange Juice, Silver, Soybeans, Sugar, Unleaded Gas, and Wheat. As for the availability of data, S\&P 500, M1, M2, Loans and Leases in Bank credit: All Commercial Bank, trade-weighted US Dollar Index and gold are gathered from historical prices or values at http://research.stlouisfed.org. The historical prices of Vanguard Total Bond Market Index Fund and Inland Real Estate Corporation are gathered at http:// finance.yahoo.com. The historical prices of Thomson Reuters / Jefferies CRB Index are downloaded at http://jefferies.com.

\section{Method of Data Analysis}

To analyze the effects of capital flow on the stock market, stock market return acts as the dependent variable while the others act as the independent variables. Firstly, I would use Pearson correlation tests to test whether each independent variable exists a linear correlation $(\rho)$ with the stock return. A t-test is conducted to verify the significance of correlation. And then the Pearson correlations will be repeated to find out whether the time-lead or time-lag of each independent variable may have a more robust correlation with the stock market return. Secondly, to explore how well the independent variables predict the return of SP500, I will be using a standard multiple regression to fit a line through a set of observations. It is a "least square" method that all set of independent variables will be entered into the equation at once. The equation is as follow:

$S P 500 \_$return $=\beta_{0}+\beta_{1} M 1$ change $+\beta_{2} M 2$ change $+\beta_{3} U S$ dollar_change $+\beta_{4}$ Bank_credit_change +

$\beta_{5}$ Bond_index_change $+\beta_{6}$ Property_return $+\beta_{7}$ Commodity_return $+\beta_{8}$ Gold_return $+\varepsilon$ 
An F-test is used to test the validity of the regression and t-tests are used to verify the significance of each variable. Finally, I will divide the data into two panels to explore the correlations with the stock market return and run regressions again. Panel A is the growth rate of M1 larger than the growth rate of M2 while Panel B is the opposite. I will expect that the predication's ability of the two panels would stronger than the original data set because one constant coefficient correlation over the whole period in the original data set is misleading which may weaken the prediction.

\section{Results}

Pearson's correlations are used to explore the linear relationship between the continuous variables. According to the guidelines suggested by Cohen (1988), the correlation of two variables is small if the coefficient is between 0.1 to 0.29 or -0.1 to -0.29 , is intermediate if the coefficient is between 0.3 to 0.49 or -0.3 to -0.49 and is large if the coefficient is between 0.5 to 1 or -0.5 to -1 . Thus there is intermediate, negative correlation between the US dollar_change and the SP500_return $(\mathrm{r}=-0.422, \mathrm{n}=443, \mathrm{p}<0.05)$, there is intermediate, positive correlation between the Commodity_return and the SP500_return $(\mathrm{r}=0.471, \mathrm{n}=443, \mathrm{p}<0.05)$ and there is small, positive correlation between the Bond_index_return and the SP500_return $(\mathrm{r}=-0.141, \mathrm{n}=443, \mathrm{p}<0.05)$. Also from the results, the M1change, M2change, and the Gold_return are positively correlated with the SP500_return while Bank_credit_change and property_return are negatively correlated with the SP500_return but they are statistically insignificant.

To investigate how well the independent variables predict the SP500_return, a standard multiple regression will be run. But to avoid the existence of multicollinearity, firstly should check that the correlation between each of the variables is not too high. Tabachnick and Fidell (2001) suggested that if the two variables have a bivariate correlation 0.7 or above, one of the variables may be needed to consider omitting or forming a composite variable from the scores of the two highly correlated variables. The highest correlation between the independent variables is -0.546 , which is less than 0.7 , therefore all variables will be retained. Moreover, regression analysis based on time series data assumes that the underlying time series is stationary. Thus, the augmented Dickey-Fuller unit root tests are conducted to test whether the time series of variables are stationary. The time series of variables are stationary.

Multicollinearity can be checked from the two values Tolerance and VIF as well. From the result presented in Table 1 labeled Coefficients, the lowest value of Tolerance is 0.606, which is not lower than 0.1 and the highest value of VIF is 1.65 , which is not higher than 10 . The two values simultaneously support that the multicollinearity does not exist in the regression. Accessing the result of the regression, from the Model Summary box, the R square is 0.314 and Adjusted R Square is 0.301. The model explains 31.4\% of the variance in the SP500_return. According to the ANOVA box, the value of Sig is 0.000 representing that the p-value is smaller than 0.05 . We will reject the null hypothesis and in favor of the alternative hypothesis meaning that the regression model reaches statistical significance. Assessing the significance of each variable from the Sig column in the Coefficients box, the values of US dollar_change, Bond_index_return, Commodity_return, and Gold_return are less than 0.05 indicating that they are making statistically significant unique contributions to predict the dependent variable while the value of other variables are higher than 0.05 meaning that they are not significant. Therefore, the regression can be written as:

SP500_return $=-0.79$ USdollar_change +0.549 Bond_index_return

+0.417 Commodity_return -0.216 Gold_return 
Table 1 Multiple Regression Result

\begin{tabular}{|c|c|c|c|c|c|c|c|c|}
\hline \multicolumn{5}{|c|}{ Model Summary $^{b}$} & & & & \\
\hline Model & $\mathrm{R}$ & R Square & $\begin{array}{l}\text { Adjusted } \\
\text { R Square } \\
\end{array}$ & $\begin{array}{l}\text { Std. Error of } \\
\text { the Estimate }\end{array}$ & & & & \\
\hline 1 & $.560^{\mathrm{a}}$ & .314 & .301 & .023442900 & & & & \\
\hline \multicolumn{9}{|c|}{$\begin{array}{l}\text { a. Predictors: (Constant), Gold_return, M1change, } \\
\text { Property_return, Bank_credit_change, Bond_index_return, } \\
\text { M2change, Commodity_return, USdollar_change } \\
\text { b. Dependent Variable: SP500_return }\end{array}$} \\
\hline \multicolumn{9}{|c|}{ ANOVA $^{a}$} \\
\hline \multicolumn{2}{|l|}{ Model } & $\begin{array}{l}\text { Sum of } \\
\text { Squares }\end{array}$ & df & $\begin{array}{l}\text { Mean } \\
\text { Square }\end{array}$ & $\mathrm{F}$ & Sig. & & \\
\hline 1 & Regression & .109 & 8 & .014 & 24.813 & $.000^{6}$ & & \\
\hline & Residual & .239 & 434 & .001 & & & & \\
\hline & Total & .348 & 442 & & & & & \\
\hline \multicolumn{9}{|c|}{ a. Dependent Variable: SP500_return } \\
\hline \multicolumn{9}{|c|}{$\begin{array}{l}\text { b. Predictors: (Constant), Gold_return, M1change, Property_return, } \\
\text { Bank_credit_change, Bond_index_return, M2change, Commodity_return, } \\
\text { USdollar_change }\end{array}$} \\
\hline \multicolumn{9}{|c|}{ Coefficients $^{\mathrm{a}}$} \\
\hline \multirow{2}{*}{\multicolumn{2}{|c|}{ Model }} & \multicolumn{2}{|c|}{$\begin{array}{c}\text { Unstandardized } \\
\text { Coefficients }\end{array}$} & $\begin{array}{l}\text { Standardized } \\
\text { Coefficients } \\
\end{array}$ & \multirow[b]{2}{*}{$\mathrm{t}$} & \multirow[b]{2}{*}{ Sig. } & \multicolumn{2}{|c|}{$\begin{array}{c}\text { Collinearity } \\
\text { Statistics } \\
\end{array}$} \\
\hline & & $\mathrm{B}$ & Std. Error & Beta & & & Tolerance & VIF \\
\hline 1 & (Constant) & .001 & .001 & & 1.067 & .286 & & \\
\hline & M1change & .015 & .112 & .006 & .132 & .895 & .894 & 1.118 \\
\hline & M2change & -.153 & .516 & -.013 & -.296 & .767 & .868 & 1.152 \\
\hline & USdollar_change & -.790 & .133 & -.303 & -5.927 & .000 & .606 & 1.650 \\
\hline & Bond_index_return & .549 & .210 & .106 & 2.612 & .009 & .967 & 1.034 \\
\hline & Bank_credit_change & -.350 & .240 & -.058 & -1.457 & .146 & .982 & 1.019 \\
\hline & Property_return & -.035 & .025 & -.055 & -1.376 & .170 & .984 & 1.017 \\
\hline & Commodity_return & .417 & .052 & .388 & 8.030 & .000 & .675 & 1.481 \\
\hline & Gold_return & -.216 & .045 & -.224 & -4.788 & .000 & .725 & 1.380 \\
\hline
\end{tabular}

However, the above analyses do not consider the lead-lag relationships between the independent variables and the dependent variable. To find out whether has a more robust correlation with the SP500_return, Table 2 repeats the Pearson Correlations using the time-lead or time-lag of each variables. Each variable will be tested time lead 1 to 13 weeks and time lag 1 to 13 weeks which will refer back to the week of 4/10/2004 and extend to 23/9/2013. For examples, M1change_lead 1 means that the change of M1 lead the return of SP500 1 week, M1change_lag 1 means that the change of M1 lag the return of SP500 1 week and so on. From the results of Table 2, all tested variables have 443 samples. For each category of variables, M1change_lag $10(\mathrm{r}=-0.109$, Sig (2-tailed) $=0.022)$, M2change_lead $1(\mathrm{r}$ $=-0.163, \operatorname{Sig}(2$-tailed $)=0.001)$, US dollar_change $(r=-0.422$, Sig $(2$-tailed $)=0.000)$, Bond_lead $1(r=-0.17$, Sig $(2-$ 
tailed $)=0.000)$, Bank_credit_lead $4(\mathrm{r}=-0.16$, Sig $(2$-tailed $)=0.001)$, Property_lead $1(\mathrm{r}=0.447$, Sig $(2$-tailed $)=$ $0.000)$, Commodity_return $(r=0.471$, Sig $(2$-tailed $)=0.000)$ and Gold_lag $3(r=0.173$, Sig $(2$-tailed $)=0.000)$ have the most robust correlations with SP500_return. Next, the ADF tests are conducted to check whether the time series of lead-lag variables are stationery. The variables USdollar_change and Commodity_return have been tested by the ADF test on the above. The time series of all lead-lag variables are stationery.

Table 3 shows the result. From the heading Collinearity Statistics in the Coefficients box, the highest Tolerance value and the lowest VIF are 0.65 and 1.539 which are not lower than 0.1 and higher than 10 respectively. Multicollinearity is not existence in the regression. In the Model Summary box, the R Square is 0.443 and Adjusted R Square is 0.433. $44.3 \%$ of the variances in the SP500_return can be predicted by the variables. The explanation of the model is better than the first model. In the ANOVA box, the value of Sig is 0.000 which is less than 0.05 . This is sufficient to conclude that there is a significant relationship between the variables and SP500_return. Assessing the significance of individual parameters, all of them are significant (Sig <0.05). Therefore, the regression equation can be written as

$S P 500$ _return $=0.003-0.272$ M1change_lag 10-1.133M2change_lead 1

-0.587USdollar_change-0.627Bond_lead1-0.641Bank_credit_lead4

+0.205 Property_lead $1+0.266$ Commodity_return +0.125 Gold_lag 3 
Table 2 Correlation between lead/lag variables and SP500_return

\begin{tabular}{|c|c|c|c|c|c|c|c|c|c|c|c|c|c|c|c|c|}
\hline & & $\begin{array}{c}\text { SP500_ } \\
\text { return }\end{array}$ & & $\begin{array}{c}\text { SP500_ } \\
\text { return }\end{array}$ & & $\begin{array}{c}\text { SP500_ } \\
\text { return }\end{array}$ & & $\begin{array}{c}\text { SP500_ } \\
\text { return }\end{array}$ & & $\begin{array}{c}\text { SP500_ } \\
\text { return }\end{array}$ & & $\begin{array}{c}\text { SP500_ } \\
\text { return }\end{array}$ & & $\begin{array}{c}\text { SP500_ } \\
\text { return }\end{array}$ & & $\begin{array}{c}\text { SP500_ } \\
\text { return }\end{array}$ \\
\hline \begin{tabular}{|l} 
Pearson \\
Correlation \\
Sig. (2- \\
tailed) \\
$\mathrm{N}$
\end{tabular} & M1change & $\begin{array}{l}.063 \\
.184 \\
443\end{array}$ & M2change & \begin{tabular}{l|}
.031 \\
.521 \\
443 \\
\end{tabular} & $\begin{array}{l}\text { USdollar_ } \\
\text { change }\end{array}$ & $\begin{array}{r}-.422^{*}= \\
.000 \\
443\end{array}$ & $\begin{array}{l}\text { Bond_ } \\
\text { index } \\
\text { return }\end{array}$ & $\begin{array}{c}.141^{37} \\
.003 \\
443\end{array}$ & $\begin{array}{l}\text { Bank__ } \\
\text { credit_ } \\
\text { change }\end{array}$ & $\begin{array}{r}-.086 \\
.071 \\
443\end{array}$ & $\begin{array}{l}\text { Property } \\
\text { return }\end{array}$ & $\begin{array}{r}-.030 \\
.524 \\
443\end{array}$ & $\begin{array}{l}\text { Commodity } \\
\text { return }\end{array}$ & $\begin{array}{r}.471^{* 7} \\
.000 \\
443\end{array}$ & $\begin{array}{l}\text { Gold_ } \\
\text { return }\end{array}$ & $\begin{array}{l}.082 \\
.086 \\
443\end{array}$ \\
\hline \begin{tabular}{|l} 
Pearson \\
Correlation \\
Sig. $(2-$ \\
tailed) \\
$\mathrm{N}$
\end{tabular} & $\begin{array}{l}\text { M1change_ } \\
\text { lead1 }\end{array}$ & $\begin{array}{r}-.004 \\
.933 \\
443\end{array}$ & $\begin{array}{l}\text { M2change } \\
\text { lead1 }\end{array}$ & $\begin{array}{r}-.163^{* *} \\
.001 \\
443\end{array}$ & $\begin{array}{l}\text { USdollar__ } \\
\text { lead1 }\end{array}$ & \begin{tabular}{r|}
.018 \\
.701 \\
443
\end{tabular} & $\begin{array}{l}\text { Bond_lea } \\
\text { d1 }\end{array}$ & $\begin{array}{r}-.170^{* *} \\
.000 \\
443\end{array}$ & $\begin{array}{l}\text { Bank_- } \\
\text { credit_ } \\
\text { lead1 }\end{array}$ & $\begin{array}{l}.002 \\
.963 \\
443\end{array}$ & $\begin{array}{l}\text { Property } \\
\text { lead1 }\end{array}$ & $\begin{array}{r}.447^{* *} \\
.000 \\
443\end{array}$ & $\begin{array}{l}\text { Commodity } \\
\text { lead1 }\end{array}$ & $\begin{array}{r}.043 \\
.369 \\
443\end{array}$ & $\begin{array}{l}\text { Gold_ } \\
\text { lead1 }\end{array}$ & $\begin{array}{r}-.075 \\
.113 \\
443\end{array}$ \\
\hline \begin{tabular}{|l} 
Pearson \\
Correlation \\
Sig. $(2-$ \\
tailed) \\
$\mathrm{N}$
\end{tabular} & $\begin{array}{l}\text { M1change_1 } \\
\text { ead2 }\end{array}$ & $\begin{array}{r}-.054 \\
.258 \\
443 \\
\end{array}$ & $\begin{array}{l}\text { M2change } \\
\text { lead2 }\end{array}$ & \begin{tabular}{l|}
.048 \\
.316 \\
443 \\
\end{tabular} & $\begin{array}{l}\text { USdollar_ } \\
\text { lead2 }\end{array}$ & \begin{tabular}{r|}
.076 \\
.109 \\
443 \\
\end{tabular} & $\begin{array}{l}\text { Bond_lea } \\
\text { d2 }\end{array}$ & $\begin{array}{l}.060 \\
.209 \\
443 \\
\end{array}$ & $\begin{array}{l}\text { Bank_- } \\
\text { credit_ } \\
\text { lead2 }\end{array}$ & $\begin{array}{r}.036 \\
.450 \\
443 \\
\end{array}$ & $\begin{array}{l}\text { Property } \\
\text { lead2 }\end{array}$ & $\begin{array}{l}.016 \\
.739 \\
443 \\
\end{array}$ & $\begin{array}{l}\text { Commodity } \\
\text { lead2 }\end{array}$ & $\begin{array}{l}.005 \\
.914 \\
443 \\
\end{array}$ & $\begin{array}{l}\text { Gold_- } \\
\text { lead2 }\end{array}$ & $\begin{array}{r}.107^{*} \\
.025 \\
443\end{array}$ \\
\hline \begin{tabular}{|l|} 
Pearson \\
Correlation \\
Sig. (2- \\
tailed) \\
$\mathrm{N}$
\end{tabular} & $\begin{array}{l}\text { M1change_ } \\
\text { lead3 }\end{array}$ & $\begin{array}{r}-.012 \\
.805 \\
443\end{array}$ & $\begin{array}{l}\text { M2change } \\
\text { lead3 }\end{array}$ & \begin{tabular}{r|}
.003 \\
.955 \\
443
\end{tabular} & $\begin{array}{l}\text { USdollar_ } \\
\text { lead3 }\end{array}$ & \begin{tabular}{r|}
$-.099^{*}$ \\
.037 \\
443
\end{tabular} & $\begin{array}{l}\text { Bond_lea } \\
\text { d3 }\end{array}$ & $\begin{array}{l}.044 \\
.355 \\
443\end{array}$ & $\begin{array}{l}\text { Bank__ } \\
\text { credit_ } \\
\text { lead3 }\end{array}$ & $\begin{array}{r}-.022 \\
.645 \\
443\end{array}$ & $\begin{array}{l}\text { Property } \\
\text { lead3 }\end{array}$ & $\begin{array}{l}.024 \\
.613 \\
443\end{array}$ & $\begin{array}{l}\text { Commodity } \\
\text { lead3 }\end{array}$ & $\begin{array}{l}.005 \\
.924 \\
443\end{array}$ & $\begin{array}{l}\text { Gold_ } \\
\text { lead3 }\end{array}$ & $\begin{array}{l}.018 \\
.698 \\
443\end{array}$ \\
\hline \begin{tabular}{|l|} 
Pearson \\
Correlation \\
Sig. (2- \\
tailed) \\
$\mathrm{N}$
\end{tabular} & $\begin{array}{l}\text { M1change_ } \\
\text { lead4 }\end{array}$ & $\begin{array}{c}-.022 \\
.637 \\
443\end{array}$ & $\begin{array}{l}\text { M2change } \\
\text { lead4 }\end{array}$ & \begin{tabular}{r|}
.015 \\
.746 \\
443
\end{tabular} & $\begin{array}{l}\text { USdollar__ } \\
\text { lead4 }\end{array}$ & \begin{tabular}{l|}
.061 \\
.201 \\
443
\end{tabular} & $\begin{array}{l}\text { Bond_lea } \\
\mathrm{d} 4\end{array}$ & $\begin{array}{r}.082 \\
.085 \\
443\end{array}$ & $\begin{array}{l}\text { Bank__ } \\
\text { credit_ } \\
\text { lead4 }\end{array}$ & $\begin{array}{r}-.160^{* 7} \\
.001 \\
443\end{array}$ & $\begin{array}{l}\text { Property } \\
\text { lead4 }\end{array}$ & $\begin{array}{l}.031 \\
.521 \\
443\end{array}$ & $\begin{array}{l}\text { Commodity } \\
\text { lead4 }\end{array}$ & $\begin{array}{r}-.025 \\
.602 \\
443\end{array}$ & \begin{tabular}{|l} 
Gold_ \\
lead4
\end{tabular} & $\begin{array}{r}-.053 \\
.263 \\
443\end{array}$ \\
\hline \begin{tabular}{|l|} 
Pearson \\
Correlation \\
Sig. (2- \\
tailed) \\
$\mathrm{N}$
\end{tabular} & $\begin{array}{l}\text { M1change_ } \\
\text { lead5 }\end{array}$ & $\begin{array}{l}.030 \\
.528 \\
443\end{array}$ & $\begin{array}{l}\text { M2change } \\
\text { lead5 }\end{array}$ & $\begin{array}{r}-.140^{* *} \\
.003 \\
443\end{array}$ & $\begin{array}{l}\text { USdollar_ } \\
\text { lead5 }\end{array}$ & \begin{tabular}{r|}
.029 \\
.539 \\
443
\end{tabular} & $\begin{array}{l}\text { Bond_lea } \\
\text { d5 }\end{array}$ & $\begin{array}{c}.169^{* *} \\
.000 \\
443\end{array}$ & $\begin{array}{l}\text { Bank_ } \\
\text { credit_ } \\
\text { lead5 }\end{array}$ & $\begin{array}{l}.097^{*} \\
.041 \\
443\end{array}$ & $\begin{array}{l}\text { Property } \\
\text { lead5 }\end{array}$ & $\begin{array}{r}-.101^{*} \\
.034 \\
443\end{array}$ & $\begin{array}{l}\text { Commodity } \\
\text { lead5 }\end{array}$ & $\begin{array}{r}-.004 \\
.937 \\
443\end{array}$ & $\begin{array}{l}\text { Gold__ } \\
\text { lead5 }\end{array}$ & $\begin{array}{r}-.021 \\
.655 \\
443\end{array}$ \\
\hline \begin{tabular}{|l|} 
Pearson \\
Correlation \\
Sig. (2- \\
tailed) \\
$\mathrm{N}$
\end{tabular} & $\begin{array}{l}\text { M1change_ } \\
\text { lead6 }\end{array}$ & $\begin{array}{l}.104^{*} \\
.029 \\
443\end{array}$ & $\begin{array}{l}\text { M2change } \\
\text { lead6 }\end{array}$ & $\begin{array}{l}.047 \\
.324 \\
443\end{array}$ & $\begin{array}{l}\text { USdollar__ } \\
\text { lead6 }\end{array}$ & \begin{tabular}{l|}
.000 \\
.999 \\
443
\end{tabular} & $\begin{array}{l}\text { Bond_lea } \\
\text { d6 }\end{array}$ & $\begin{array}{l}.054 \\
.255 \\
443\end{array}$ & $\begin{array}{l}\text { Bank_- } \\
\text { credit_ } \\
\text { lead6 }\end{array}$ & $\begin{array}{r}-.116^{*} \\
.014 \\
443\end{array}$ & $\begin{array}{l}\text { Property } \\
\text { lead6 }\end{array}$ & $\begin{array}{r}.146^{* *} \\
.002 \\
443\end{array}$ & $\begin{array}{l}\text { Commodity } \\
\text { lead6 }\end{array}$ & $\begin{array}{l}.048 \\
.313 \\
443\end{array}$ & $\begin{array}{l}\text { Gold__ } \\
\text { lead6 }\end{array}$ & $\begin{array}{l}.050 \\
.293 \\
443\end{array}$ \\
\hline \begin{tabular}{|l|} 
Pearson \\
Correlation \\
Sig. (2- \\
tailed) \\
$\mathrm{N}$
\end{tabular} & $\begin{array}{l}\text { M1chnage } \\
\text { lead7 }\end{array}$ & $\begin{array}{r}-.077 \\
.108 \\
443\end{array}$ & $\begin{array}{l}\text { M2change } \\
\text { lead7 }\end{array}$ & \begin{tabular}{l|}
.023 \\
.632 \\
443
\end{tabular} & $\begin{array}{l}\text { USdollar_ } \\
\text { lead7 }\end{array}$ & \begin{tabular}{r|}
.088 \\
.064 \\
443
\end{tabular} & $\begin{array}{l}\text { Bond_lea } \\
\text { d7 }\end{array}$ & $\begin{array}{r}-.088 \\
.064 \\
443\end{array}$ & $\begin{array}{l}\text { Bank__ } \\
\text { credit_ } \\
\text { lead7 }\end{array}$ & $\begin{array}{r}.077 \\
.104 \\
443\end{array}$ & $\begin{array}{l}\text { Property } \\
\text { lead7 }\end{array}$ & $\begin{array}{r}-.030 \\
.528 \\
443\end{array}$ & $\begin{array}{l}\text { Commodity } \\
\text { lead7 }\end{array}$ & \begin{tabular}{l|}
.039 \\
.411 \\
443
\end{tabular} & $\begin{array}{l}\text { Gold__ } \\
\text { lead7 }\end{array}$ & $\begin{array}{l}.058 \\
.224 \\
443\end{array}$ \\
\hline \begin{tabular}{|l|} 
Pearson \\
Correlation \\
Sig. (2- \\
tailed) \\
$\mathrm{N}$
\end{tabular} & $\begin{array}{l}\text { M1change_ } \\
\text { lead8 }\end{array}$ & $\begin{array}{l}.006 \\
.907 \\
443\end{array}$ & $\begin{array}{l}\text { M2change } \\
\text { lead8 }\end{array}$ & \begin{tabular}{r|}
.030 \\
.532 \\
443
\end{tabular} & $\begin{array}{l}\text { USdollar_ } \\
\text { lead8 }\end{array}$ & \begin{tabular}{r|}
-.030 \\
.524 \\
443
\end{tabular} & $\begin{array}{l}\text { Bond_lea } \\
\mathrm{d} 8\end{array}$ & $\begin{array}{l}.040 \\
.399 \\
443\end{array}$ & $\begin{array}{l}\text { Bank_- } \\
\text { credit_ } \\
\text { lead8 }\end{array}$ & $\begin{array}{r}.091 \\
.057 \\
443\end{array}$ & $\begin{array}{l}\text { Property } \\
\text { lead8 }\end{array}$ & $\begin{array}{l}.039 \\
.417 \\
443\end{array}$ & $\begin{array}{l}\text { Commodity } \\
\text { lead8 }\end{array}$ & $\begin{array}{r}.131^{* *} \\
.006 \\
443\end{array}$ & $\begin{array}{l}\text { Gold__ } \\
\text { lead8 }\end{array}$ & $\begin{array}{l}.004 \\
.928 \\
443\end{array}$ \\
\hline \begin{tabular}{|l} 
Pearson \\
Correlation \\
Sig. (2- \\
tailed) \\
$\mathrm{N}$
\end{tabular} & $\begin{array}{l}\text { M1change_ } \\
\text { lead9 }\end{array}$ & $\begin{array}{r}-.029 \\
.539 \\
443\end{array}$ & $\begin{array}{l}\text { M2chnage } \\
\text { lead9 }\end{array}$ & \begin{tabular}{r|}
$.108^{*}$ \\
.023 \\
443
\end{tabular} & $\begin{array}{l}\text { USdollar__ } \\
\text { lead9 }\end{array}$ & \begin{tabular}{r|}
$.106^{*}$ \\
.026 \\
443
\end{tabular} & $\begin{array}{l}\text { Bond_lea } \\
\text { d9 }\end{array}$ & \begin{tabular}{r|}
$.117^{*}$ \\
.014 \\
443
\end{tabular} & $\begin{array}{l}\text { Bank_- } \\
\text { credit_ } \\
\text { lead99 }\end{array}$ & \begin{tabular}{r|}
.008 \\
.869 \\
443
\end{tabular} & $\begin{array}{l}\text { Property } \\
\text { lead9 }\end{array}$ & $\begin{array}{r}-.027 \\
.569 \\
443\end{array}$ & $\begin{array}{l}\text { Commodity } \\
\text { lead9 }\end{array}$ & $\begin{array}{r}-.142^{* *} \\
.003 \\
443\end{array}$ & $\begin{array}{l}\text { Gold_ } \\
\text { lead9 }\end{array}$ & $\begin{array}{r}-.062 \\
.193 \\
443\end{array}$ \\
\hline \begin{tabular}{|l|} 
Pearson \\
Correlation \\
Sig. (2- \\
tailed) \\
$\mathrm{N}$
\end{tabular} & $\begin{array}{l}\text { M1change_ } \\
\text { lead10 }\end{array}$ & $\begin{array}{l}.006 \\
.905 \\
443\end{array}$ & $\begin{array}{l}\text { M2change } \\
\text { lead10 }\end{array}$ & $\begin{array}{l}.028 \\
.550 \\
443\end{array}$ & $\begin{array}{l}\text { USdollar_ } \\
\text { lead10 }\end{array}$ & $\begin{array}{l}.070 \\
.143 \\
443\end{array}$ & $\begin{array}{l}\text { Bond_lea } \\
\text { d10 }\end{array}$ & $\begin{array}{l}.052 \\
.278 \\
443\end{array}$ & $\begin{array}{l}\text { Bank_- } \\
\text { credit_ } \\
\text { lead10 }\end{array}$ & $\begin{array}{r}-.023 \\
.629 \\
443\end{array}$ & $\begin{array}{l}\text { Property } \\
\text { lead10 }\end{array}$ & $\begin{array}{r}-.084 \\
.078 \\
443\end{array}$ & $\begin{array}{l}\text { Commodity_ } \\
\text { lead10 }\end{array}$ & $\begin{array}{l}.020 \\
.671 \\
443\end{array}$ & $\begin{array}{l}\text { Gold__ } \\
\text { lead10 }\end{array}$ & $\begin{array}{l}.062 \\
.194 \\
443\end{array}$ \\
\hline \begin{tabular}{|l|} 
Pearson \\
Correlation \\
Sig. (2- \\
tailed) \\
$\mathrm{N}$
\end{tabular} & $\begin{array}{l}\text { M1change_ } \\
\text { lead11 }\end{array}$ & $\begin{array}{r}-.042 \\
.374 \\
443\end{array}$ & $\begin{array}{l}\text { M2change } \\
\text { lead11 }\end{array}$ & \begin{tabular}{r|}
$.120^{*}$ \\
.011 \\
443
\end{tabular} & $\begin{array}{l}\text { USdollar_ } \\
\text { lead11 }\end{array}$ & $\begin{array}{r}-.065 \\
.170 \\
443\end{array}$ & $\begin{array}{l}\text { Bond_lea } \\
\text { d11 }\end{array}$ & $\begin{array}{r}-.009 \\
.846 \\
443\end{array}$ & $\begin{array}{l}\text { Bank_- } \\
\text { credit_ } \\
\text { lead11 }\end{array}$ & $\begin{array}{r}-.020 \\
.674 \\
443\end{array}$ & $\begin{array}{l}\text { Property } \\
\text { lead11 }\end{array}$ & $\begin{array}{r}-.098^{*} \\
.038 \\
443\end{array}$ & $\begin{array}{l}\text { Commodity } \\
\text { lead11 }\end{array}$ & $\begin{array}{r}.157^{* *} \\
.001 \\
443\end{array}$ & $\begin{array}{l}\text { Gold_ } \\
\text { lead11 }\end{array}$ & $\begin{array}{l}.014 \\
.765 \\
443\end{array}$ \\
\hline \begin{tabular}{|l|} 
Pearson \\
Correlation \\
Sig. (2- \\
tailed) \\
$\mathrm{N}$
\end{tabular} & $\begin{array}{l}\text { M1change_ } \\
\text { lead12 }\end{array}$ & $\begin{array}{r}-.097^{*} \\
.041 \\
443\end{array}$ & $\begin{array}{l}\text { M2change } \\
\text { lead12 }\end{array}$ & \begin{tabular}{r|}
. .038 \\
.424 \\
443
\end{tabular} & $\begin{array}{l}\text { USdollar__ } \\
\text { lead12 }\end{array}$ & \begin{tabular}{r|}
-.014 \\
.773 \\
443
\end{tabular} & $\begin{array}{l}\text { Bond_lea } \\
\text { d12 }\end{array}$ & $\begin{array}{r}-.070 \\
.140 \\
443\end{array}$ & $\begin{array}{l}\text { Bank_ } \\
\text { credit_ } \\
\text { lead12 }\end{array}$ & $\begin{array}{r}.003 \\
.951 \\
443\end{array}$ & $\begin{array}{l}\text { Property } \\
\text { lead12 }\end{array}$ & $\begin{array}{r}-.060 \\
.208 \\
443\end{array}$ & $\begin{array}{l}\text { Commodity } \\
\text { lead12 }\end{array}$ & $\begin{array}{r}-.006 \\
.897 \\
443\end{array}$ & $\begin{array}{l}\text { Gold__ } \\
\text { lead12 }\end{array}$ & $\begin{array}{l}.035 \\
.458 \\
443\end{array}$ \\
\hline \begin{tabular}{|l|} 
Pearson \\
Correlation \\
Sig. (2- \\
tailed) \\
$\mathrm{N}$
\end{tabular} & $\begin{array}{l}\text { M1change_ } \\
\text { lead13 }\end{array}$ & $\begin{array}{l}.013 \\
.786 \\
443\end{array}$ & $\begin{array}{l}\text { M2change } \\
\text { lead13 }\end{array}$ & \begin{tabular}{r|}
.034 \\
.475 \\
443
\end{tabular} & $\begin{array}{l}\text { USdollar__ } \\
\text { lead13 }\end{array}$ & \begin{tabular}{r|}
-.030 \\
.526 \\
443
\end{tabular} & $\begin{array}{l}\text { Bond_lea } \\
\text { d13 }\end{array}$ & $\begin{array}{r}.021 \\
.663 \\
443\end{array}$ & $\begin{array}{l}\text { Bank_ } \\
\text { credit_ } \\
\text { lead13 }\end{array}$ & $\begin{array}{r}-.041 \\
.387 \\
443\end{array}$ & $\begin{array}{l}\text { Property } \\
\text { lead13 }\end{array}$ & $\begin{array}{r}-.070 \\
.139 \\
443\end{array}$ & $\begin{array}{l}\text { Commodity } \\
\text { lead13 }\end{array}$ & \begin{tabular}{l|}
.021 \\
.662 \\
443
\end{tabular} & $\begin{array}{l}\text { Gold__ } \\
\text { lead13 }\end{array}$ & $\begin{array}{l}.033 \\
.489\end{array}$ \\
\hline \begin{tabular}{|l|} 
Pearson \\
Correlation \\
Sig. (2- \\
tailed) \\
$\mathrm{N}$
\end{tabular} & $\begin{array}{l}\text { M1change_ } \\
\text { lag1 }\end{array}$ & $\begin{array}{r}-.081 \\
.087 \\
443\end{array}$ & $\begin{array}{l}\text { M2change } \\
\text { lag1 }\end{array}$ & \begin{tabular}{r|}
.083 \\
.082 \\
443
\end{tabular} & $\begin{array}{l}\text { USdollar } \\
\text { lag1 }\end{array}$ & \begin{tabular}{r|}
-.033 \\
.485 \\
443
\end{tabular} & $\begin{array}{l}\text { Bond_lag } \\
1\end{array}$ & $\begin{array}{l}.051 \\
.287 \\
443\end{array}$ & $\begin{array}{l}\text { Bank_ } \\
\text { credit_ } \\
\text { lag1 }\end{array}$ & $\begin{array}{r}-.002 \\
.971 \\
443\end{array}$ & $\begin{array}{l}\text { Property_ } \\
\text { lag1 }\end{array}$ & $\begin{array}{r}.124^{2 *} \\
.009 \\
443\end{array}$ & $\begin{array}{l}\text { Commodity_ } \\
\text { lag1 }\end{array}$ & $\begin{array}{r}-.003 \\
.945 \\
443\end{array}$ & $\begin{array}{l}\text { Gold_1 } \\
\text { ag1 }\end{array}$ & $\begin{array}{l}.027 \\
.568 \\
443\end{array}$ \\
\hline $\begin{array}{l}\text { Pearson } \\
\text { Correlation } \\
\text { Sig. }(2- \\
\text { tailed) } \\
\mathrm{N}\end{array}$ & $\begin{array}{l}\text { M1change_ } \\
\text { lag2 }\end{array}$ & $\begin{array}{l}.026 \\
.585 \\
443\end{array}$ & $\begin{array}{l}\text { M2change } \\
\text { lag2 }\end{array}$ & \begin{tabular}{r|}
. $.121^{*}$ \\
.011 \\
443
\end{tabular} & $\begin{array}{l}\text { USdollar_ } \\
\text { lag2 }\end{array}$ & $\begin{array}{l}.039 \\
.407 \\
443\end{array}$ & $\begin{array}{l}\text { Bond_lag } \\
2\end{array}$ & $\begin{array}{r}-.057 \\
.231 \\
443\end{array}$ & $\begin{array}{l}\text { Bank_- } \\
\text { credit_ } \\
\text { lag2 }\end{array}$ & $\begin{array}{r}-.036 \\
.448 \\
443\end{array}$ & $\begin{array}{l}\text { Property } \\
\text { lag2 }\end{array}$ & $\begin{array}{r}-.024 \\
.614 \\
443\end{array}$ & $\begin{array}{l}\text { Commodity } \\
\text { lag2 }\end{array}$ & $\begin{array}{r}-.030 \\
.527 \\
443\end{array}$ & $\begin{array}{l}\text { Gold_1 } \\
\text { ag2 }\end{array}$ & $\begin{array}{r}-.082 \\
.087 \\
443\end{array}$ \\
\hline $\begin{array}{l}\text { Pearson } \\
\text { Correlation }\end{array}$ & $\begin{array}{l}\text { M1change_ } \\
\text { lag3 }\end{array}$ & -.002 & $\begin{array}{l}\text { M2change_ } \\
\text { lag3 }\end{array}$ & -.038 & $\begin{array}{l}\text { USdollar_ } \\
\text { lag3 }\end{array}$ & -.080 & $\begin{array}{l}\text { Bond_lag } \\
3\end{array}$ & .079 & $\begin{array}{l}\text { Bank_- } \\
\text { credit_ }\end{array}$ & .084 & $\begin{array}{l}\text { Property_ } \\
\text { lag3 }\end{array}$ & .063 & $\begin{array}{l}\text { Commodity } \\
\text { lag3 }\end{array}$ & $.153^{* *}$ & $\begin{array}{l}\text { Gold_1 } \\
\text { ag3 }\end{array}$ & $.173^{* *}$ \\
\hline
\end{tabular}




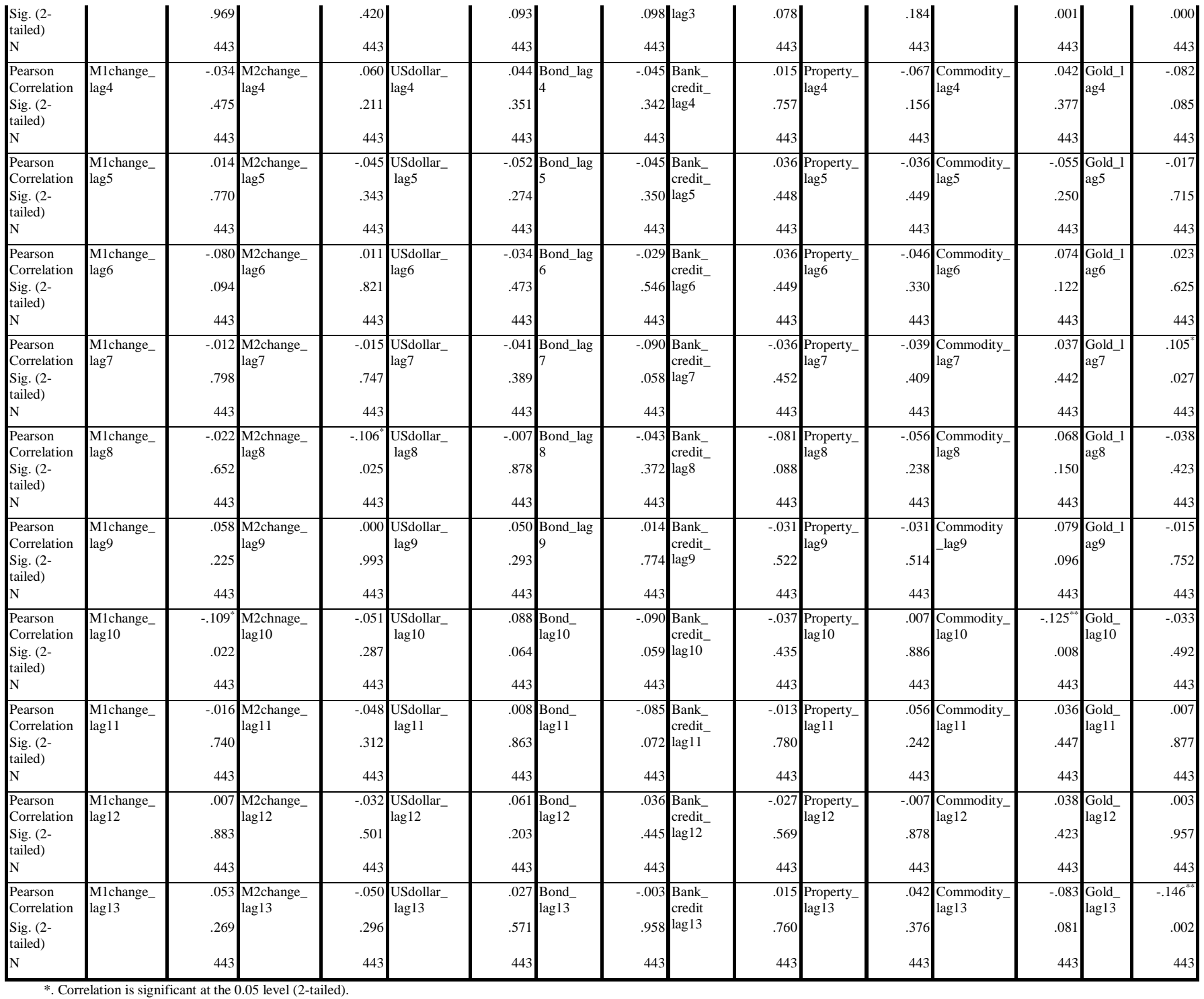

**. Correlation is significant at the 0.01 level (2-tailed)

Table 3 Multiple Regression Result with lead / lag dependent variables

\begin{tabular}{|c|c|c|c|c|c|c|c|}
\hline \multicolumn{8}{|c|}{ Model Summary } \\
\hline \multirow[b]{2}{*}{ Model } & \multirow[b]{2}{*}{$\mathrm{R}$} & \multirow[b]{2}{*}{ R Square } & \multirow[b]{2}{*}{$\begin{array}{l}\text { Adjusted } \\
\text { R Square }\end{array}$} & \multirow[b]{2}{*}{$\begin{array}{l}\text { Std. Error of } \\
\text { the Estimate }\end{array}$} & \multicolumn{2}{|c|}{ Change Statistics } & \multirow[b]{2}{*}{$\begin{array}{l}\text { Durbin- } \\
\text { Watson }\end{array}$} \\
\hline & & & & & $\begin{array}{c}\text { R } \\
\text { Square } \\
\text { Change }\end{array}$ & $\begin{array}{c}\mathrm{F} \\
\text { Change }\end{array}$ & \\
\hline 1 & $.666^{2}$ & .443 & .433 & .021121178 & .443 & 43.150 & 2.331 \\
\hline
\end{tabular}

a. Predictors: (Constant), M1change_lag10, USdollar_change, M2change_lead1,

Bank_credit_lead4, Gold_lag3, Bond_lead1, Property_lead1, Commodity_return

b. Dependent Variable: SP500_return 


\begin{tabular}{|c|c|c|c|c|c|c|c|c|}
\hline \multicolumn{7}{|c|}{ ANOVA $^{a}$} & & \\
\hline \multicolumn{2}{|c|}{ Model } & \begin{tabular}{|c|} 
Sum of \\
Squares
\end{tabular} & df & Mean Square & $\mathrm{F}$ & Sig. & & \\
\hline \multirow[t]{3}{*}{1} & Regression & .154 & 8 & .019 & 43.150 & $.000^{6}$ & & \\
\hline & Residual & .194 & 434 & .000 & & & & \\
\hline & Total & .348 & 442 & & & & & \\
\hline \multicolumn{9}{|c|}{ a. Dependent Variable: SP500_return } \\
\hline \multicolumn{9}{|c|}{$\begin{array}{l}\text { b. Predictors: (Constant), M1change_lag10, USdollar_change, M2change_lead1, } \\
\text { Bank_credit_lead4, Gold_lag3, Bond_lead1, Property_lead1, Commodity_return }\end{array}$} \\
\hline \multicolumn{9}{|c|}{ Coefficients $^{\mathrm{a}}$} \\
\hline \multirow{2}{*}{\multicolumn{2}{|c|}{ Model }} & \multicolumn{2}{|c|}{$\begin{array}{l}\text { Unstandardized } \\
\text { Coefficients }\end{array}$} & \begin{tabular}{c|} 
Standardized \\
Coefficients
\end{tabular} & & & \multicolumn{2}{|c|}{ Collinearity Statistics } \\
\hline & & $\mathrm{B}$ & $\begin{array}{l}\text { Std. } \\
\text { Error }\end{array}$ & Beta & $\mathrm{t}$ & Sig. & Tolerance & VIF \\
\hline \multirow[t]{9}{*}{1} & (Constant) & .003 & .001 & & 2.552 & .011 & & \\
\hline & USdollar_change & -.587 & .115 & -.225 & -5.086 & .000 & .655 & 1.527 \\
\hline & Commodity_return & .266 & .048 & .247 & 5.567 & .000 & .650 & 1.539 \\
\hline & Bond_lead1 & -.627 & .193 & -.121 & -3.251 & .001 & .933 & 1.072 \\
\hline & Bank_credit_lead4 & -.641 & .215 & -.108 & -2.978 & .003 & .984 & 1.017 \\
\hline & Property_lead1 & .205 & .023 & .324 & 8.741 & .000 & .933 & 1.072 \\
\hline & Gold_lag3 & .125 & .035 & .130 & 3.589 & .000 & .974 & 1.026 \\
\hline & M2change_lead1 & -1.133 & .442 & -.093 & -2.563 & .011 & .964 & 1.037 \\
\hline & M1change_lag10 & -.272 & .096 & -.102 & -2.819 & .005 & .982 & 1.018 \\
\hline
\end{tabular}

The sufficiency of funds in the investment market plays an important role in the relationship between independent variables and SP500_return. It can be affected by different points of view from investors, policies adopted by regulators and the availability of fund in different economic circumstances (Li 2007). For examples, the positive views for the economy in the late 1990s encouraged investments, investors were willing to invest and the fund of the market was sufficient, the correlation between assets is somehow positive. But when the stock market crashed in 2008, investors became risk-averse and held more "safety" assets such as gold and bonds, Hartmann et al. (2001) called this phenomenon "flight to quality". On the other hand, when the market recovery, investors may switch their investment to those high return again. This phenomenon is known as "flight from quality". In these two situations, the correlations between the return of the stock market and "safety" assets became negative. Simultaneously, the strength of the correlations between SP500_retrun and independent variables may change or even have conflicts over the whole period. A constant correlation coefficient is misleading. In order to decrease the above effect on the predictions of independent variables, I divide the data into two panels. Panel A is when the growth rate of M1 larger than the growth rate of M2 representing that the fund is sufficient while Panel B is when the growth rate of M2 larger than the growth rate of M1 representing that the fund is not sufficient in the investment market. From the original data set, 236 out of 443 samples are the growth rate of M1 greater than the growth of M2 while the other 207 samples are the growth rate of M2 greater than the growth rate of M1. Thus, Panel A has 236 samples and Panel B has 207 samples. Person correlations are tested for the lead-lag relation of independent variables and SP500_return in the two panels. 
The results of Panel A as Table 4 shows, M1change_lead 12 ( r=-0.186, Sig (2-tailed) = 0.004), M2change_lead 1 ( r=0.266 , Sig $(2$-tailed $)=0.000)$, US dollar_change $(r=-0.396$, Sig $(2$-tailed $)=0.000)$ Bond_lead $4(\mathrm{r}=-0.205$, Sig $(2-$ tailed $)=0.000)$, Bank_credit_lead $4(\mathrm{r}=-0.323$, Sig (2-tailed) $=0.000)$, Property_lead $1(\mathrm{r}=0.469$, Sig $(2$-tailed $)=$ $0.000)$, Commodity_return $(\mathrm{r}=0.427$, Sig $(2$-tailed $)=0.000)$ and Gold_lag $3(\mathrm{r}=0.299$, Sig $(2$-tailed $)=0.000)$ are the most correlated variables with SP500_return in each category. For the results of Panel B as shown in Table 5, M1change_lead 7 ( $\mathrm{r}=-0.19$, Sig (2-tailed) = 0.006), M2change_lag $2(\mathrm{r}=-0.221$, Sig (2-tailed $)=0.001)$, US dollar_change $(r=-0.459$, Sig $(2$-tailed $)=0.000)$, Bond_lead $1(r=-0.249$, Sig (2-tailed $)=0.000)$, Bank_credit_lead 7 ( $\mathrm{r}=-0.167, \operatorname{Sig}(2$-tailed $)=0.016)$, Property_lead $1(\mathrm{r}=0.424$, Sig $(2$-tailed $)=0.000)$, Commodity_return $(\mathrm{r}=0.526$, Sig $(2$-tailed $)=0.000)$ and Gold_lead $2(\mathrm{r}=-0.181$, Sig $(2$-tailed $)=0.009)$ are the most correlated variables. One point to mention is that the highest coefficient correlation of gold with SP_500 return is positive in Panel A and is negative in Panel B.

Table 4 Correlation between lead/lag variables and SP500_return (Panel A)

\begin{tabular}{|c|c|c|c|c|c|c|c|c|c|c|c|c|c|c|c|c|}
\hline & & $\begin{array}{c}\text { SP500_ } \\
\text { return }\end{array}$ & & $\begin{array}{c}\text { SP500_ } \\
\text { return }\end{array}$ & & $\begin{array}{c}\text { SP500_ } \\
\text { return }\end{array}$ & & $\begin{array}{c}\text { SP500_ } \\
\text { return }\end{array}$ & & $\begin{array}{c}\text { SP500_ } \\
\text { return }\end{array}$ & & $\begin{array}{c}\text { SP500_ } \\
\text { return }\end{array}$ & & $\begin{array}{c}\text { SP500_ } \\
\text { return }\end{array}$ & & $\begin{array}{c}\text { SP500_ } \\
\text { return }\end{array}$ \\
\hline $\begin{array}{l}\text { Pearson } \\
\text { Correlation } \\
\text { Sig. (2- } \\
\text { tailed) } \\
\mathrm{N} \\
\end{array}$ & M1change & $\begin{array}{l}.122 \\
.061 \\
236 \\
\end{array}$ & M2change & $\begin{array}{l}.045 \\
.493 \\
236 \\
\end{array}$ & $\begin{array}{l}\text { USdollar_ } \\
\text { change }\end{array}$ & $\begin{array}{r}-.396 \\
.000 \\
236 \\
\end{array}$ & $\begin{array}{l}\text { Bond_ } \\
\text { index_- } \\
\text { return }\end{array}$ & $\begin{array}{l}.148^{\circ} \\
\\
.023 \\
236 \\
\end{array}$ & $\begin{array}{l}\text { Bank_- } \\
\text { credit_- } \\
\text { change }\end{array}$ & $\begin{array}{r}.206 * \\
.001 \\
236 \\
\end{array}$ & $\begin{array}{l}\text { Property } \\
\text { return }\end{array}$ & $\begin{array}{r}-.128^{*} \\
.049 \\
236 \\
\end{array}$ & $\begin{array}{l}\text { Commodity }_{-} \\
\text {return }\end{array}$ & $\begin{array}{l}.427^{* 2} \\
.000 \\
236\end{array}$ & $\begin{array}{l}\text { Gold_ } \\
\text { return }\end{array}$ & $\begin{array}{l}.091 \\
.165 \\
236\end{array}$ \\
\hline $\begin{array}{l}\text { Pearson } \\
\text { Correlation } \\
\text { Sig. (2- } \\
\text { tailed) } \\
\text { N }\end{array}$ & $\begin{array}{l}\text { M1change_ } \\
\text { lead1 }\end{array}$ & $\begin{array}{c}-.090 \\
.166 \\
236\end{array}$ & $\begin{array}{l}\text { M2change } \\
\text { lead1 }\end{array}$ & $\begin{array}{r}-.266^{* *} \\
.000 \\
236\end{array}$ & $\begin{array}{l}\text { USdollar_ } \\
\text { lead1 }\end{array}$ & $\begin{array}{l}.084 \\
.197 \\
236\end{array}$ & $\begin{array}{l}\text { Bond_lea } \\
\text { d1 }\end{array}$ & $\begin{array}{r}-.098 \\
.135 \\
236\end{array}$ & $\begin{array}{l}\text { Bank_- } \\
\text { credit__ } \\
\text { lead1 }\end{array}$ & $\begin{array}{r}-.001 \\
.989 \\
236\end{array}$ & $\begin{array}{l}\text { Property } \\
\text { lead1 }\end{array}$ & $\begin{array}{c}.469^{* * 7} \\
.000 \\
236\end{array}$ & $\begin{array}{l}\text { Commodity } \\
\text { lead1 }\end{array}$ & $\begin{array}{r}-.132 \\
.043 \\
236\end{array}$ & $\begin{array}{l}\text { Gold_ } \\
\text { lead1 }\end{array}$ & $\begin{array}{r}-.079 \\
.225 \\
236\end{array}$ \\
\hline $\begin{array}{l}\text { Pearson } \\
\text { Correlation } \\
\text { Sig. (2- } \\
\text { tailed) } \\
\mathrm{N}\end{array}$ & $\begin{array}{l}\text { M1change_1 } \\
\text { ead2 }\end{array}$ & $\begin{array}{c}-.047 \\
.471 \\
236\end{array}$ & $\begin{array}{l}\text { M2change_ } \\
\text { lead2 }\end{array}$ & $\begin{array}{l}.079 \\
.226 \\
236\end{array}$ & $\begin{array}{l}\text { USdollar_ } \\
\text { lead2 }\end{array}$ & $\begin{array}{c}-.021 \\
.752 \\
236\end{array}$ & $\begin{array}{l}\text { Bond_lea } \\
\text { d2 }\end{array}$ & $\begin{array}{l}.124 \\
.058 \\
236\end{array}$ & $\begin{array}{l}\text { Bank_- } \\
\text { credit_- } \\
\text { lead2 }\end{array}$ & $\begin{array}{c}-.050 \\
.448 \\
236\end{array}$ & $\begin{array}{l}\text { Property_ } \\
\text { lead2 }\end{array}$ & $\begin{array}{l}.066 \\
.315 \\
236\end{array}$ & $\begin{array}{l}\text { Commodity } \\
\text { lead2 }\end{array}$ & $\begin{array}{l}.080 \\
.222 \\
236\end{array}$ & $\begin{array}{l}\text { Gold__ } \\
\text { lead2 }\end{array}$ & $\begin{array}{c}-.045 \\
.492 \\
236\end{array}$ \\
\hline $\begin{array}{l}\text { Pearson } \\
\text { Correlation } \\
\text { Sig. (2- } \\
\text { tailed) } \\
\mathrm{N}\end{array}$ & $\begin{array}{l}\text { M1change_ } \\
\text { lead3 }\end{array}$ & $\begin{array}{l}.009 \\
.888 \\
236\end{array}$ & $\begin{array}{l}\text { M2change_ } \\
\text { lead3 }\end{array}$ & $\begin{array}{l}.077 \\
.241 \\
236\end{array}$ & $\begin{array}{l}\text { USdollar_ } \\
\text { lead3 }\end{array}$ & $\begin{array}{r}-.123 \\
.060 \\
236\end{array}$ & $\begin{array}{l}\text { Bond_lea } \\
\text { d3 }\end{array}$ & $\begin{array}{l}.045 \\
.496 \\
236\end{array}$ & $\begin{array}{l}\text { Bank_- } \\
\text { credit__ } \\
\text { lead3 }\end{array}$ & $\begin{array}{l}.004 \\
.957 \\
236\end{array}$ & $\begin{array}{l}\text { Property_ } \\
\text { lead3 }\end{array}$ & $\begin{array}{l}.076 \\
.246 \\
236\end{array}$ & $\begin{array}{l}\text { Commodity } \\
\text { lead3 }\end{array}$ & $\begin{array}{l}.025 \\
.704 \\
236\end{array}$ & $\begin{array}{l}\text { Gold__ } \\
\text { lead3 }\end{array}$ & $\begin{array}{r}-.022 \\
.741 \\
236\end{array}$ \\
\hline $\begin{array}{l}\text { Pearson } \\
\text { Correlation } \\
\text { Sig. (2- } \\
\text { tailed) } \\
\mathrm{N}\end{array}$ & $\begin{array}{l}\text { M1change_ } \\
\text { lead4 }\end{array}$ & $\begin{array}{c}-.014 \\
.830 \\
236\end{array}$ & $\begin{array}{l}\text { M2change_ } \\
\text { lead4 }\end{array}$ & $\begin{array}{l}.008 \\
.897 \\
236\end{array}$ & $\begin{array}{l}\text { USdollar_ } \\
\text { lead4 }\end{array}$ & $\begin{array}{l}.096 \\
.140 \\
236\end{array}$ & $\begin{array}{l}\text { Bond_lea } \\
\text { d4 }\end{array}$ & $\begin{array}{r}-.205^{* *} \\
.002 \\
236\end{array}$ & $\begin{array}{l}\text { Bank_- } \\
\text { credit_ } \\
\text { lead4 }\end{array}$ & $\begin{array}{r}-.323^{* *} \\
.000 \\
236\end{array}$ & $\begin{array}{l}\text { Property_ } \\
\text { lead4 }\end{array}$ & $\begin{array}{l}.077 \\
.236 \\
236\end{array}$ & $\begin{array}{l}\text { Commodity } \\
\text { lead4 }\end{array}$ & $\begin{array}{r}-.052 \\
.422 \\
236\end{array}$ & $\begin{array}{l}\text { Gold__ } \\
\text { lead4 }\end{array}$ & $\begin{array}{r}-.068 \\
.301 \\
236\end{array}$ \\
\hline $\begin{array}{l}\text { Pearson } \\
\text { Correlation } \\
\text { Sig. (2- } \\
\text { tailed) } \\
\mathrm{N}\end{array}$ & $\begin{array}{l}\text { M1change_ } \\
\text { lead5 }\end{array}$ & $\begin{array}{l}.005 \\
.940 \\
236\end{array}$ & $\begin{array}{l}\text { M2change_ } \\
\text { lead5 }\end{array}$ & $\begin{array}{r}-.189^{* 7} \\
.004 \\
236\end{array}$ & $\begin{array}{l}\text { USdollar_ } \\
\text { lead5 }\end{array}$ & $\begin{array}{l}.088 \\
.177 \\
236\end{array}$ & $\begin{array}{l}\text { Bond_lea } \\
\text { d5 }\end{array}$ & $\begin{array}{r}.188^{* 7} \\
.004 \\
236\end{array}$ & $\begin{array}{l}\text { Bank_- } \\
\text { credit_ } \\
\text { lead5 }\end{array}$ & $\begin{array}{l}.105 \\
.107 \\
236\end{array}$ & \begin{tabular}{|l|} 
Property_ \\
lead5
\end{tabular} & $\begin{array}{r}-.175^{* 2} \\
.007 \\
236\end{array}$ & $\begin{array}{l}\text { Commodity_ } \\
\text { lead5 }\end{array}$ & $\begin{array}{r}-.193^{* * 2} \\
.003 \\
236\end{array}$ & $\begin{array}{l}\text { Gold__ } \\
\text { lead5 }\end{array}$ & $\begin{array}{r}-.088 \\
.176 \\
236\end{array}$ \\
\hline $\begin{array}{l}\text { Pearson } \\
\text { Correlation } \\
\text { Sig. (2- } \\
\text { tailed) } \\
\mathrm{N}\end{array}$ & $\begin{array}{l}\text { M1change_ } \\
\text { lead6 }\end{array}$ & $\begin{array}{l}.082 \\
.209 \\
236\end{array}$ & $\begin{array}{l}\text { M2change_ } \\
\text { lead6 }\end{array}$ & $\begin{array}{l}.070 \\
.285 \\
236\end{array}$ & $\begin{array}{l}\text { USdollar_ } \\
\text { lead6 }\end{array}$ & $\begin{array}{r}-.034 \\
.608 \\
236\end{array}$ & $\begin{array}{l}\text { Bond_lea } \\
\text { d6 }\end{array}$ & $\begin{array}{l}.117 \\
.072 \\
236\end{array}$ & $\begin{array}{l}\text { Bank_- } \\
\text { credit__ } \\
\text { lead6 }\end{array}$ & $\begin{array}{r}.098 \\
.132 \\
236\end{array}$ & $\begin{array}{l}\text { Property } \\
\text { lead6 }\end{array}$ & $\begin{array}{r}.179^{* 7} \\
.006 \\
236\end{array}$ & $\begin{array}{l}\text { Commodity } \\
\text { lead6 }\end{array}$ & $\begin{array}{l}.052 \\
.425 \\
236\end{array}$ & $\begin{array}{l}\text { Gold_ } \\
\text { lead6 }\end{array}$ & $\begin{array}{l}.098 \\
.134 \\
236\end{array}$ \\
\hline $\begin{array}{l}\text { Pearson } \\
\text { Correlation } \\
\text { Sig. (2- } \\
\text { tailed) } \\
\text { N }\end{array}$ & $\begin{array}{l}\text { M1chnage_ } \\
\text { lead7 }\end{array}$ & $\begin{array}{c}.004 \\
.947 \\
236\end{array}$ & $\begin{array}{l}\text { M2change_ } \\
\text { lead7 }\end{array}$ & $\begin{array}{l}.131^{*} \\
.044 \\
236\end{array}$ & $\begin{array}{l}\text { USdollar_ } \\
\text { lead7 }\end{array}$ & $\begin{array}{l}.107 \\
.102 \\
236\end{array}$ & $\begin{array}{l}\text { Bond_lea } \\
\text { d7 }\end{array}$ & $\begin{array}{r}-.062 \\
.346 \\
236\end{array}$ & $\begin{array}{l}\text { Bank_- } \\
\text { credit_- } \\
\text { lead7 }\end{array}$ & $\begin{array}{l}.042 \\
.521 \\
236\end{array}$ & \begin{tabular}{|l} 
Property_ \\
lead7
\end{tabular} & $\begin{array}{r}-.162 \\
.013 \\
236\end{array}$ & $\begin{array}{l}\text { Commodity_ } \\
\text { lead7 }\end{array}$ & $\begin{array}{l}.013 \\
.846 \\
236\end{array}$ & $\begin{array}{l}\text { Gold__ } \\
\text { lead7 }\end{array}$ & $\begin{array}{l}.027 \\
.679 \\
236\end{array}$ \\
\hline $\begin{array}{l}\text { Pearson } \\
\text { Correlation } \\
\text { Sig. (2- } \\
\text { tailed) } \\
\text { N }\end{array}$ & $\begin{array}{l}\text { M1change_ } \\
\text { lead8 }\end{array}$ & $\begin{array}{r}-.013 \\
.844 \\
236\end{array}$ & $\begin{array}{l}\text { M2change_ } \\
\text { lead8 }\end{array}$ & $\begin{array}{l}.010 \\
.873 \\
236\end{array}$ & $\begin{array}{l}\text { USdollar_ } \\
\text { lead8 }\end{array}$ & $\begin{array}{r}-.044 \\
.503 \\
236\end{array}$ & $\begin{array}{l}\text { Bond_lea } \\
\text { d8 }\end{array}$ & $\begin{array}{l}.037 \\
.575 \\
236\end{array}$ & $\begin{array}{l}\text { Bank_ } \\
\text { credit_- } \\
\text { lead8 }\end{array}$ & $\begin{array}{r}-.139^{*} \\
.033 \\
236\end{array}$ & $\begin{array}{l}\text { Property_ } \\
\text { lead8 }\end{array}$ & $\begin{array}{l}.097 \\
.136 \\
236\end{array}$ & $\begin{array}{l}\text { Commodity_ } \\
\text { lead8 }\end{array}$ & $\begin{array}{l}.119 \\
.068 \\
236\end{array}$ & $\begin{array}{l}\text { Gold__ } \\
\text { lead8 }\end{array}$ & $\begin{array}{l}.121 \\
.063 \\
236\end{array}$ \\
\hline $\begin{array}{l}\text { Pearson } \\
\text { Correlation } \\
\text { Sig. (2- } \\
\text { tailed) } \\
\text { N }\end{array}$ & $\begin{array}{l}\text { M1change_ } \\
\text { lead9 }\end{array}$ & $\begin{array}{c}.022 \\
.742 \\
236\end{array}$ & $\begin{array}{l}\text { M2chnage_ } \\
\text { lead9 }\end{array}$ & $\begin{array}{r}-.131^{*} \\
.045 \\
236\end{array}$ & $\begin{array}{l}\text { USdollar_ } \\
\text { lead9 }\end{array}$ & $\begin{array}{l}.120 \\
.066 \\
236\end{array}$ & $\begin{array}{l}\text { Bond_lea } \\
\text { d9 }\end{array}$ & $\begin{array}{r}-.174^{* *} \\
.007 \\
236\end{array}$ & $\begin{array}{l}\text { Bank_- } \\
\text { credit_- } \\
\text { lead9 }\end{array}$ & $\begin{array}{l}.054 \\
.408 \\
236\end{array}$ & \begin{tabular}{|l} 
Property_ \\
lead9
\end{tabular} & $\begin{array}{l}.121 \\
.064 \\
236\end{array}$ & $\begin{array}{l}\text { Commodity_ } \\
\text { lead9 }\end{array}$ & $\begin{array}{r}-.176^{* *} \\
.007 \\
236\end{array}$ & $\begin{array}{l}\text { Gold_ } \\
\text { lead9 }\end{array}$ & $\begin{array}{r}.104 \\
.110 \\
236\end{array}$ \\
\hline $\begin{array}{l}\text { Pearson } \\
\text { Correlation } \\
\text { Sig. (2- } \\
\text { tailed) } \\
\mathrm{N}\end{array}$ & $\begin{array}{l}\text { M1change_ } \\
\text { lead10 }\end{array}$ & $\begin{array}{l}.031 \\
.639 \\
236\end{array}$ & $\begin{array}{l}\text { M2change_ } \\
\text { lead10 }\end{array}$ & $\begin{array}{r}.017 \\
.800 \\
236\end{array}$ & $\begin{array}{l}\text { USdollar_ } \\
\text { lead10 }\end{array}$ & $\begin{array}{l}.036 \\
.582 \\
236\end{array}$ & $\begin{array}{l}\text { Bond_lea } \\
\text { d10 }\end{array}$ & $\begin{array}{l}.070 \\
.287 \\
236\end{array}$ & $\begin{array}{l}\text { Bank_- } \\
\text { credit_- } \\
\text { lead10 }\end{array}$ & $\begin{array}{c}-.006 \\
.922 \\
236\end{array}$ & $\begin{array}{l}\text { Property } \\
\text { lead10 }\end{array}$ & $\begin{array}{r}-.029 \\
.654 \\
236\end{array}$ & $\begin{array}{l}\text { Commodity_ } \\
\text { lead10 }\end{array}$ & \begin{tabular}{r|}
-.026 \\
.694 \\
236
\end{tabular} & $\begin{array}{l}\text { Gold__ } \\
\text { lead10 }\end{array}$ & $\begin{array}{l}.179^{n+7} \\
.006 \\
236\end{array}$ \\
\hline $\begin{array}{l}\text { Pearson } \\
\text { Correlation } \\
\text { Sig. (2- } \\
\text { tailed) } \\
\text { N }\end{array}$ & $\begin{array}{l}\text { M1change_ } \\
\text { lead11 }\end{array}$ & $\begin{array}{l}.027 \\
.680 \\
236\end{array}$ & $\begin{array}{l}\text { M2change_ } \\
\text { lead11 }\end{array}$ & $\begin{array}{r}.181^{* *} \\
.005 \\
236\end{array}$ & $\begin{array}{l}\text { USdollar_ } \\
\text { lead11 }\end{array}$ & $\begin{array}{r}.008 \\
.902 \\
236\end{array}$ & $\begin{array}{l}\text { Bond_lea } \\
\text { d11 }\end{array}$ & $\begin{array}{r}.028 \\
.664 \\
236\end{array}$ & $\begin{array}{l}\text { Bank_} \\
\text { credit_ } \\
\text { lead11 }\end{array}$ & $\begin{array}{l}.044 \\
.505 \\
236\end{array}$ & $\begin{array}{l}\text { Property_ } \\
\text { lead11 }\end{array}$ & $\begin{array}{l}.101 \\
.122 \\
236\end{array}$ & $\begin{array}{l}\text { Commodity } \\
\text { lead11 }\end{array}$ & $\begin{array}{l}.132^{*} \\
.042 \\
236\end{array}$ & $\begin{array}{l}\text { Gold_ } \\
\text { lead11 }\end{array}$ & $\begin{array}{r}.025 \\
.699 \\
236\end{array}$ \\
\hline \begin{tabular}{|l} 
Pearson \\
Correlation \\
Sig. (2- \\
tailed) \\
$\mathrm{N}$
\end{tabular} & $\begin{array}{l}\text { M1change_ } \\
\text { lead12 }\end{array}$ & $\begin{array}{r}-.186^{*} \\
.004 \\
236\end{array}$ & $\begin{array}{l}\text { M2change } \\
\text { lead12 }\end{array}$ & $\begin{array}{c}-.051 \\
.433 \\
236\end{array}$ & $\begin{array}{l}\text { USdollar_ } \\
\text { lead12 }\end{array}$ & $\begin{array}{l}.062 \\
.346 \\
236\end{array}$ & $\begin{array}{l}\text { Bond_lea } \\
\text { d12 }\end{array}$ & $\begin{array}{c}-.023 \\
.728 \\
236\end{array}$ & $\begin{array}{l}\text { Bank_} \\
\text { credit_- } \\
\text { lead12 }\end{array}$ & $\begin{array}{l}.042 \\
.522 \\
236\end{array}$ & $\begin{array}{l}\text { Property } \\
\text { lead12 }\end{array}$ & $\begin{array}{c}-.044 \\
.502 \\
236\end{array}$ & $\begin{array}{l}\text { Commodity } \\
\text { lead12 }\end{array}$ & \begin{tabular}{r|}
.095 \\
.146 \\
236
\end{tabular} & $\begin{array}{l}\text { Gold_ } \\
\text { lead12 }\end{array}$ & $\begin{array}{l}.001 \\
.990 \\
236\end{array}$ \\
\hline
\end{tabular}




\begin{tabular}{|c|c|c|c|c|c|c|c|c|c|c|c|c|c|c|c|c|}
\hline $\begin{array}{l}\text { Pearson } \\
\text { Correlation } \\
\text { Sig. (2- } \\
\text { tailed) } \\
\mathrm{N}\end{array}$ & $\mid \begin{array}{l}\text { M1change__ } \\
\text { lead13 }\end{array}$ & $\begin{array}{r}-.005 \\
.944 \\
236 \\
\end{array}$ & $\begin{array}{l}\text { M2change } \\
\text { lead13 }\end{array}$ & $\begin{array}{r}-.074 \\
.258 \\
236 \\
\end{array}$ & $\begin{array}{l}\text { USdollar_ } \\
\text { lead13 }\end{array}$ & $\begin{array}{l}.049 \\
.450 \\
236 \\
\end{array}$ & $\mid \begin{array}{l}\text { Bond_lea } \\
\mathrm{d} 13\end{array}$ & $\begin{array}{r}-.130^{*} \\
.046 \\
236 \\
\end{array}$ & $\begin{array}{l}\text { Bank_ } \\
\text { credit_- } \\
\text { lead13 }\end{array}$ & \begin{tabular}{r|}
-.044 \\
.499 \\
236 \\
\end{tabular} & $\begin{array}{l}\text { Property } \\
\text { lead13 }\end{array}$ & $\begin{array}{r}-.103 \\
.114 \\
236 \\
\end{array}$ & $\mid \begin{array}{l}\text { Commodity_ } \\
\text { lead13 }\end{array}$ & $\begin{array}{c}-.009 \\
.895 \\
236 \\
\end{array}$ & $\begin{array}{l}\text { Gold_- } \\
\text { lead13 }\end{array}$ & $\begin{array}{r}-.019 \\
.767\end{array}$ \\
\hline $\begin{array}{l}\text { Pearson } \\
\text { Correlation } \\
\text { Sig. (2- } \\
\text { tailed) } \\
\mathrm{N}\end{array}$ & $\begin{array}{l}\text { M1change } \\
\text { lag1 }\end{array}$ & $\begin{array}{r}-.123 \\
.059 \\
236\end{array}$ & $\begin{array}{l}\text { M2change_ } \\
\text { lag1 }\end{array}$ & $\begin{array}{r}-.045 \\
.494 \\
236\end{array}$ & $\begin{array}{l}\begin{array}{l}\text { USdollar_ } \\
\text { lag1 }\end{array} \\
\text { lat }\end{array}$ & $\begin{array}{l}.068 \\
.295 \\
236\end{array}$ & $\begin{array}{l}\text { Bond_lag } \\
1\end{array}$ & $\begin{array}{l}.085 \\
.192 \\
236\end{array}$ & $\begin{array}{l}\text { Bank_ } \\
\text { credit_ } \\
\text { lag1 }\end{array}$ & \begin{tabular}{l|}
.033 \\
.610 \\
236
\end{tabular} & \begin{tabular}{|l} 
Property \\
lag1
\end{tabular} & $\begin{array}{l}.079 \\
.226 \\
236\end{array}$ & \begin{tabular}{|l|} 
Commodity \\
lag1
\end{tabular} & $\begin{array}{c}. .099 \\
.129 \\
236\end{array}$ & \begin{tabular}{|l} 
Gold_1 \\
ag1
\end{tabular} & 171 \\
\hline $\begin{array}{l}\text { Pearson } \\
\text { Correlation } \\
\text { Sig. (2- } \\
\text { tailed) } \\
\mathrm{N}\end{array}$ & $\begin{array}{l}\text { M1change } \\
\text { lag2 }\end{array}$ & \begin{tabular}{l|}
.068 \\
.296 \\
236
\end{tabular} & $\begin{array}{l}\text { M2change } \\
\text { lag2 }\end{array}$ & $\begin{array}{l}.044 \\
.502 \\
236\end{array}$ & $\begin{array}{l}\text { USdollar_ } \\
\text { lag2 }\end{array}$ & $\begin{array}{l}.052 \\
.425 \\
236\end{array}$ & $\begin{array}{l}\text { Bond_lag } \\
2\end{array}$ & $\begin{array}{l}.008 \\
.902 \\
236\end{array}$ & 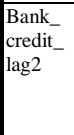 & \begin{tabular}{c|}
-.041 \\
.528 \\
236
\end{tabular} & \begin{tabular}{|l|}
$\begin{array}{l}\text { Property } \\
\text { lag22 }\end{array}$ \\
\end{tabular} & \begin{tabular}{r|}
-.094 \\
.150 \\
236
\end{tabular} & \begin{tabular}{|l|} 
Commodity \\
lag2
\end{tabular} & $\begin{array}{c}. .074 \\
.259 \\
236\end{array}$ & \begin{tabular}{|l|} 
Gold_1 \\
ag2
\end{tabular} & -.17 \\
\hline $\begin{array}{l}\text { Pearson } \\
\text { Correlation } \\
\text { Sig. (2- } \\
\text { tailed) } \\
\mathrm{N}\end{array}$ & \begin{tabular}{|l|} 
M1change \\
lag3
\end{tabular} & $\begin{array}{r}-.010 \\
.879 \\
236\end{array}$ & $\begin{array}{l}\text { M2change } \\
\text { lag3 }\end{array}$ & $\begin{array}{l}.040 \\
.537 \\
236\end{array}$ & \begin{tabular}{|l|} 
USdollar_ \\
lag3
\end{tabular} & $\begin{array}{c}.139 \\
.033 \\
236\end{array}$ & \begin{tabular}{|l|} 
Bond_lag \\
3
\end{tabular} & \begin{tabular}{l|}
.067 \\
.309 \\
236
\end{tabular} & $\begin{array}{l}\begin{array}{l}\text { Bank_ } \\
\text { credit_ } \\
\text { lag33 }\end{array} \\
\end{array}$ & \begin{tabular}{l|}
.115 \\
.079 \\
236
\end{tabular} & \begin{tabular}{|l|} 
Property \\
lag3
\end{tabular} & $\begin{array}{c}.151 \\
.020 \\
236\end{array}$ & \begin{tabular}{|l|} 
Commodity \\
lag3
\end{tabular} & $\begin{array}{c}.172^{*} \\
.008 \\
236\end{array}$ & \begin{tabular}{|l|} 
Gold_1 \\
ag3
\end{tabular} & \\
\hline $\begin{array}{l}\text { Pearson } \\
\text { Correlation } \\
\text { Sig. (2- } \\
\text { tailed) } \\
\mathrm{N}\end{array}$ & \begin{tabular}{|l|} 
M1change \\
lag4
\end{tabular} & $\begin{array}{l}.051 \\
.440 \\
236\end{array}$ & $\begin{array}{l}\text { M2change_ } \\
\text { lag4 }\end{array}$ & $\begin{array}{l}.137^{\circ} \\
.035 \\
236\end{array}$ & $\begin{array}{l}\text { USdollar_ } \\
\text { lag4 }\end{array}$ & \begin{tabular}{r|}
-.054 \\
.407 \\
236
\end{tabular} & $\begin{array}{l}\text { Bond_lag } \\
4\end{array}$ & \begin{tabular}{r|}
.082 \\
.207 \\
236
\end{tabular} & $\begin{array}{l}\begin{array}{l}\text { Bank_ } \\
\text { credit_ } \\
\text { lag44 }\end{array} \\
\end{array}$ & \begin{tabular}{l|}
.004 \\
.948 \\
236
\end{tabular} & \begin{tabular}{|l} 
Property \\
lag4
\end{tabular} & \begin{tabular}{r|}
-.103 \\
.114 \\
236
\end{tabular} & \begin{tabular}{|l|} 
Commodity \\
lag4
\end{tabular} & $\begin{array}{c}.136 \\
.037 \\
236\end{array}$ & \begin{tabular}{|l|} 
Gold_l \\
ag4
\end{tabular} & \\
\hline $\begin{array}{l}\text { Pearson } \\
\text { Correlation } \\
\text { Sig. (2- } \\
\text { tailed) } \\
\mathrm{N}\end{array}$ & \begin{tabular}{|l|} 
M1change \\
lag5
\end{tabular} & \begin{tabular}{l|}
.024 \\
.712 \\
236
\end{tabular} & $\begin{array}{l}\text { M2change } \\
\text { lag5 }\end{array}$ & $\begin{array}{l}.019 \\
.767 \\
236\end{array}$ & $\begin{array}{l}\text { USdollar_ } \\
\text { lag55 }\end{array}$ & \begin{tabular}{r|}
-.134 \\
.039 \\
236
\end{tabular} & $\begin{array}{l}\text { Bond_lag } \\
5\end{array}$ & $\begin{array}{l}.034 \\
.602 \\
236\end{array}$ & \begin{tabular}{|l}
$\begin{array}{l}\text { Bank } \\
\text { creditit } \\
\text { lag55 }\end{array}$ \\
\end{tabular} & \begin{tabular}{r|}
-.004 \\
.953 \\
236
\end{tabular} & \begin{tabular}{|l|} 
Property \\
lag55
\end{tabular} & \begin{tabular}{r|}
-.065 \\
.318 \\
236
\end{tabular} & \begin{tabular}{|l|} 
Commodity \\
lag5
\end{tabular} & \begin{tabular}{r|}
-.071 \\
.279 \\
236
\end{tabular} & $\begin{array}{l}\text { Gold_1 } \\
\text { ag5 }\end{array}$ & \\
\hline $\begin{array}{l}\text { Pearson } \\
\text { Correlation } \\
\text { Sig. (2- } \\
\text { tailed) } \\
\mathrm{N}\end{array}$ & $\begin{array}{l}\text { M1change _ } \\
\text { lag6 }\end{array}$ & \begin{tabular}{c|}
-.091 \\
.163 \\
236
\end{tabular} & $\begin{array}{l}\text { M2change } \\
\text { lag6 }\end{array}$ & $\begin{array}{l}.018 \\
.780 \\
236\end{array}$ & $\begin{array}{l}\text { USdollar_ } \\
\text { lag6 }\end{array}$ & \begin{tabular}{c|}
-.028 \\
.666 \\
236
\end{tabular} & $\begin{array}{l}\text { Bond_lag } \\
6\end{array}$ & \begin{tabular}{l|}
.033 \\
.609 \\
236
\end{tabular} & $\begin{array}{l}\text { Bank_- } \\
\text { credit_- } \\
\text { lag6 }\end{array}$ & \begin{tabular}{c|}
.019 \\
.767 \\
236
\end{tabular} & \begin{tabular}{|l}
$\begin{array}{l}\text { Property } \\
\text { lag66 }\end{array}$ \\
\end{tabular} & $\begin{array}{r}.036 \\
.584 \\
236\end{array}$ & $\begin{array}{l}\text { Commodity } \\
\text { lag6 }\end{array}$ & \begin{tabular}{l|}
.099 \\
.131 \\
236
\end{tabular} & $\begin{array}{l}\text { Gold_1 } \\
\text { ag6 }\end{array}$ & \\
\hline $\begin{array}{l}\text { Pearson } \\
\text { Correlation } \\
\text { Sig. (2- } \\
\text { tailed) } \\
\mathrm{N}\end{array}$ & $\begin{array}{l}\text { M1change } \\
\text { lag7 }\end{array}$ & \begin{tabular}{r|}
-.040 \\
.546 \\
236
\end{tabular} & $\begin{array}{l}\text { M2change } \\
\text { lag7 }\end{array}$ & $\begin{array}{r}.023 \\
.726 \\
236\end{array}$ & $\begin{array}{l}\text { USdollar_ } \\
\text { lag7 }\end{array}$ & $\begin{array}{l}.023 \\
.724 \\
236\end{array}$ & $\begin{array}{l}\text { Bond_lag } \\
7\end{array}$ & \begin{tabular}{r|}
$-.145^{*}$ \\
.025 \\
236
\end{tabular} & $\begin{array}{l}\begin{array}{l}\text { Bank_- } \\
\text { credit_- } \\
\text { lag7 }\end{array} \\
\end{array}$ & \begin{tabular}{r|}
-.080 \\
.220 \\
236
\end{tabular} & \begin{tabular}{|l} 
Property \\
lag7
\end{tabular} & \begin{tabular}{r|}
$.145^{3}$ \\
.026 \\
236
\end{tabular} & $\begin{array}{l}\text { Commodity } \\
\text { lag7 }\end{array}$ & \begin{tabular}{l|}
.071 \\
.277 \\
236
\end{tabular} & \begin{tabular}{|l|} 
Gold_1 \\
ag7
\end{tabular} & \\
\hline $\begin{array}{l}\text { Pearson } \\
\text { Correlation } \\
\text { Sig. (2- } \\
\text { tailed) } \\
\mathrm{N}\end{array}$ & \begin{tabular}{|l|} 
Mlchange \\
lag8
\end{tabular} & $\begin{array}{c}-.006 \\
.924 \\
236\end{array}$ & $\begin{array}{l}\text { M2chnage_ } \\
\text { lag8 }\end{array}$ & $\begin{array}{l}.045 \\
.487 \\
236\end{array}$ & \begin{tabular}{|l|} 
USdollar_ \\
lag8
\end{tabular} & \begin{tabular}{l|}
.011 \\
.862 \\
236
\end{tabular} & $\begin{array}{l}\text { Bond_lag } \\
8\end{array}$ & $\begin{array}{r}-.043 \\
.508 \\
236\end{array}$ & $\begin{array}{l}\begin{array}{l}\text { Bank_ } \\
\text { credit_ } \\
\text { lag8 }\end{array} \\
\end{array}$ & \begin{tabular}{c|}
-.112 \\
.085 \\
236
\end{tabular} & \begin{tabular}{|l} 
Property \\
lag8
\end{tabular} & $\begin{array}{r}-.042 \\
.519 \\
236\end{array}$ & $\begin{array}{l}\text { Commodity } \\
\text { lag8 }\end{array}$ & \begin{tabular}{l|}
.098 \\
.134 \\
236
\end{tabular} & $\begin{array}{l}\text { Gold_1 } \\
\text { ag8 }\end{array}$ & \\
\hline $\begin{array}{l}\text { Pearson } \\
\text { Correlation } \\
\text { Sig. (2- } \\
\text { tailed) } \\
\mathrm{N}\end{array}$ & \begin{tabular}{|l|} 
M1change \\
lag9
\end{tabular} & $\begin{array}{l}.094 \\
.148 \\
236\end{array}$ & $\begin{array}{l}\text { M2change_ } \\
\text { lag9 }\end{array}$ & $\begin{array}{l}.066 \\
.316 \\
236\end{array}$ & \begin{tabular}{|l|} 
USdollar_ \\
lag99
\end{tabular} & $\begin{array}{l}.004 \\
.953 \\
236\end{array}$ & $\begin{array}{l}\text { Bond_lag } \\
9\end{array}$ & \begin{tabular}{l|}
.029 \\
.658 \\
236
\end{tabular} & $\begin{array}{l}\text { Bank_- } \\
\text { credit_- } \\
\text { lag9 }\end{array}$ & \begin{tabular}{l|}
.097 \\
.139 \\
236
\end{tabular} & \begin{tabular}{|l|} 
Property \\
lag9
\end{tabular} & $\begin{array}{c}.087 \\
.183 \\
236\end{array}$ & \begin{tabular}{|l|} 
Commodity \\
lag9
\end{tabular} & \begin{tabular}{l|}
.091 \\
.162 \\
236
\end{tabular} & \begin{tabular}{|l|} 
Gold_l \\
ag9
\end{tabular} & 10 \\
\hline $\begin{array}{l}\text { Pearson } \\
\text { Correlation } \\
\text { Sig. (2- } \\
\text { tailed) } \\
\mathrm{N}\end{array}$ & \begin{tabular}{|l} 
M1change \\
lag10
\end{tabular} & \begin{tabular}{r|}
$.149^{\prime}$ \\
.022 \\
236
\end{tabular} & $\begin{array}{l}\text { M2chnage_ } \\
\text { lag10 }\end{array}$ & $\begin{array}{r}.074 \\
.255 \\
236\end{array}$ & \begin{tabular}{|l|} 
USdollar_ \\
lag10
\end{tabular} & $\begin{array}{l}.044 \\
.500 \\
236\end{array}$ & $\begin{array}{l}\text { Bond__ } \\
\text { lag10 }\end{array}$ & \begin{tabular}{r|}
$-.154^{*}$ \\
.018 \\
236
\end{tabular} & \begin{tabular}{|l}
$\begin{array}{l}\text { Bank_- } \\
\text { credit_- } \\
\text { lag10 }\end{array}$ \\
\end{tabular} & \begin{tabular}{c|}
-.064 \\
.326 \\
236
\end{tabular} & \begin{tabular}{|l|} 
Property \\
lag10
\end{tabular} & $\begin{array}{l}.050 \\
.449 \\
236\end{array}$ & $\begin{array}{l}\text { Commodity } \\
\text { lag10 }\end{array}$ & \begin{tabular}{r|}
-.161 \\
.013 \\
236
\end{tabular} & $\begin{array}{l}\text { Gold_- } \\
\text { lag10 }\end{array}$ & 103 \\
\hline $\begin{array}{l}\text { Pearson } \\
\text { Correlation } \\
\text { Sig. (2- } \\
\text { tailed) } \\
\mathrm{N}\end{array}$ & $\begin{array}{l}\text { M1change_ } \\
\text { lag11 }\end{array}$ & $\begin{array}{c}-.030 \\
.644 \\
236\end{array}$ & $\begin{array}{l}\text { M2change } \\
\text { lag11 }\end{array}$ & $\begin{array}{r}-.082 \\
.208 \\
236\end{array}$ & $\begin{array}{l}\text { USdollar_ } \\
\text { lag11 }\end{array}$ & $\begin{array}{l}.026 \\
.691 \\
236\end{array}$ & $\begin{array}{l}\text { Bond__ } \\
\text { lag11 }\end{array}$ & \begin{tabular}{r|}
-.108 \\
.097 \\
236
\end{tabular} & $\begin{array}{l}\text { Bank_- } \\
\text { credit_- } \\
\text { lag11 }\end{array}$ & \begin{tabular}{c|}
-.049 \\
.458 \\
236
\end{tabular} & \begin{tabular}{|l|} 
Property \\
lag11
\end{tabular} & $\begin{array}{r}-.057 \\
.384 \\
236\end{array}$ & \begin{tabular}{|l} 
Commodity \\
lag11
\end{tabular} & $\begin{array}{l}.033 \\
.616 \\
236\end{array}$ & $\begin{array}{l}\text { Gold_- } \\
\text { lag11 }\end{array}$ & 13 \\
\hline $\begin{array}{l}\text { Pearson } \\
\text { Correlation } \\
\text { Sig. (2- } \\
\text { tailed) } \\
\mathrm{N}\end{array}$ & \begin{tabular}{|l|} 
M1change \\
lag12
\end{tabular} & \begin{tabular}{r|}
.009 \\
.894 \\
236
\end{tabular} & $\begin{array}{l}\text { M2change } \\
\text { lag12 }\end{array}$ & $\begin{array}{r}.097 \\
.136 \\
236\end{array}$ & 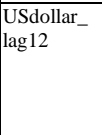 & \begin{tabular}{l|}
.061 \\
.351 \\
236
\end{tabular} & \begin{tabular}{|l} 
Bond_- \\
lag12
\end{tabular} & \begin{tabular}{l|}
.029 \\
.658 \\
236
\end{tabular} & $\begin{array}{l}\begin{array}{l}\text { Bank } \\
\text { credit_ } \\
\text { lag12 }\end{array} \\
\text { lage }\end{array}$ & \begin{tabular}{c|}
-.022 \\
.734 \\
236
\end{tabular} & \begin{tabular}{|l|}
$\begin{array}{l}\text { Property } \\
\text { lag12 }\end{array}$ \\
\end{tabular} & $\begin{array}{r}.012 \\
.856 \\
236\end{array}$ & \begin{tabular}{|l} 
Commodity \\
lag12
\end{tabular} & $\begin{array}{l}.094 \\
.149 \\
236\end{array}$ & 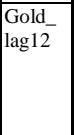 & 105 \\
\hline $\begin{array}{l}\text { Pearson } \\
\text { Correlation } \\
\text { Sig. (2- } \\
\text { tailed) } \\
\mathrm{N}\end{array}$ & \begin{tabular}{|l} 
Mlchange \\
lag13
\end{tabular} & $\begin{array}{l}.072 \\
.270 \\
236\end{array}$ & $\begin{array}{l}\text { M2change } \\
\text { lag13 }\end{array}$ & $\begin{array}{l}.061 \\
.349 \\
236\end{array}$ & \begin{tabular}{|l|} 
USdollar \\
lag13
\end{tabular} & \begin{tabular}{l|}
.023 \\
.723 \\
236
\end{tabular} & \begin{tabular}{|l} 
Bond_- \\
lag13
\end{tabular} & $\begin{array}{r}.036 \\
.581 \\
236\end{array}$ & \begin{tabular}{|l|}
$\begin{array}{l}\text { Bank } \\
\text { credit } \\
\text { lag13 }\end{array}$ \\
\end{tabular} & $\begin{array}{r}.015 \\
.819 \\
-.206^{* *}\end{array}$ & \begin{tabular}{|l|} 
Property \\
lag13
\end{tabular} & $\begin{array}{l}.030 \\
.646 \\
236\end{array}$ & \begin{tabular}{|l|} 
Commodity \\
$\operatorname{lag} 13$
\end{tabular} & $\begin{array}{r}.132^{*} \\
.042 \\
236\end{array}$ & $\begin{array}{l}\text { Gold_- } \\
\operatorname{lag} 13\end{array}$ & 7 \\
\hline
\end{tabular}

**. Correlation is significant at the 0.01 level (2-tailed). 
Table 5 Correlation between lead/lag variables and SP500_return (Panel B)

\begin{tabular}{|c|c|c|c|c|c|c|c|c|c|c|c|c|c|c|c|c|}
\hline & & $\begin{array}{c}\mathrm{SP} 500_{-} \\
\text {return }\end{array}$ & & $\begin{array}{c}\text { SP500_ } \\
\text { return }\end{array}$ & & $\begin{array}{c}\text { SP500_ } \\
\text { return }\end{array}$ & & $\begin{array}{l}\text { SP500_ } \\
\text { return }\end{array}$ & & $\begin{array}{c}\text { SP500_ } \\
\text { return }\end{array}$ & & $\begin{array}{c}\mathrm{SP} 500 \\
\text { return }\end{array}$ & & $\begin{array}{l}\text { SP500_ } \\
\text { return }\end{array}$ & & $\begin{array}{c}\text { SP500_ } \\
\text { return }\end{array}$ \\
\hline $\begin{array}{l}\text { Pearson } \\
\text { Correlation } \\
\text { Sig. (2- } \\
\text { tailed) } \\
\mathrm{N}\end{array}$ & M1change & $\begin{array}{l}.034 \\
.626 \\
207\end{array}$ & M2change & $\begin{array}{l}.006 \\
.936 \\
207\end{array}$ & $\begin{array}{l}\text { USdollar_ } \\
\text { change }\end{array}$ & $\begin{array}{r}-.457^{* *} \\
.000 \\
207\end{array}$ & $\begin{array}{l}\text { Bond_ } \\
\text { index_ } \\
\text { return }\end{array}$ & $\begin{array}{l}.132 \\
.058 \\
207\end{array}$ & $\begin{array}{l}\text { Bank__ } \\
\text { credit_- } \\
\text { change }\end{array}$ & $\begin{array}{l}.010 \\
.884 \\
207\end{array}$ & $\begin{array}{l}\text { Property } \\
\text { return }\end{array}$ & $\begin{array}{l}.113 \\
.105 \\
207\end{array}$ & $\begin{array}{l}\text { Commodity } \\
\text { return }\end{array}$ & $\begin{array}{c}.526^{* 7} \\
.000 \\
207\end{array}$ & $\begin{array}{l}\text { Gold_ } \\
\text { return }\end{array}$ & $\begin{array}{l}.069 \\
.324 \\
207\end{array}$ \\
\hline \begin{tabular}{|l} 
Pearson \\
Correlation \\
Sig. (2- \\
tailed) \\
$\mathrm{N}$
\end{tabular} & $\begin{array}{l}\text { M1change_ } \\
\text { lead1 }\end{array}$ & $\begin{array}{l}.084 \\
.228 \\
207\end{array}$ & $\begin{array}{l}\text { M2change_ } \\
\text { lead1 }\end{array}$ & \begin{tabular}{l|}
.005 \\
.944 \\
207
\end{tabular} & $\begin{array}{l}\text { USdollar_ } \\
\text { lead1 }\end{array}$ & $\begin{array}{r}-.066 \\
.348 \\
207\end{array}$ & $\begin{array}{l}\text { Bond_lea } \\
\text { d1 }\end{array}$ & $\begin{array}{r}-.249^{* *} \\
.000 \\
207\end{array}$ & $\begin{array}{l}\text { Bank__ } \\
\text { credit_ } \\
\text { lead1 }\end{array}$ & $\begin{array}{l}.004 \\
.950 \\
207\end{array}$ & $\begin{array}{l}\text { Property } \\
\text { lead1 }\end{array}$ & $\begin{array}{r}.424^{* *} \\
.000 \\
207\end{array}$ & $\begin{array}{l}\text { Commodity } \\
\text { lead1 }\end{array}$ & $\begin{array}{l}.067 \\
.338 \\
207\end{array}$ & $\begin{array}{l}\text { Gold__ } \\
\text { lead1 }\end{array}$ & $\begin{array}{r}-.069 \\
.320 \\
207\end{array}$ \\
\hline $\begin{array}{l}\text { Pearson } \\
\text { Correlation } \\
\text { Sig. }(2- \\
\text { tailed) } \\
\mathrm{N}\end{array}$ & $\begin{array}{l}\text { M1change_1 } \\
\text { ead2 }\end{array}$ & $\begin{array}{r}-.064 \\
.363 \\
207\end{array}$ & $\begin{array}{l}\text { M2change_ } \\
\text { lead2 }\end{array}$ & $\begin{array}{l}.016 \\
.819 \\
207\end{array}$ & $\begin{array}{l}\text { USdollar__ } \\
\text { lead2 }\end{array}$ & $\begin{array}{r}.203^{* *} \\
.003 \\
207\end{array}$ & $\begin{array}{l}\text { Bond_lea } \\
\text { d2 }\end{array}$ & $\begin{array}{r}-.011 \\
.878 \\
207\end{array}$ & $\begin{array}{l}\text { Bank__ } \\
\text { credit_ } \\
\text { lead2 }\end{array}$ & $\begin{array}{r}-.013 \\
.855 \\
207\end{array}$ & $\begin{array}{l}\text { Property } \\
\text { lead2 }\end{array}$ & $\begin{array}{r}-.041 \\
.554 \\
207\end{array}$ & $\begin{array}{l}\text { Commodity } \\
\text { lead2 }\end{array}$ & $\begin{array}{c}-.081 \\
.248 \\
207\end{array}$ & $\begin{array}{l}\text { Gold__ } \\
\text { lead2 }\end{array}$ & $\begin{array}{r}-.181^{* *} \\
.009 \\
207\end{array}$ \\
\hline \begin{tabular}{|l|} 
Pearson \\
Correlation \\
Sig. (2- \\
tailed) \\
$\mathrm{N}$
\end{tabular} & $\begin{array}{l}\text { M1change_ } \\
\text { lead3 }\end{array}$ & $\begin{array}{r}-.042 \\
.546 \\
207\end{array}$ & $\begin{array}{l}\text { M2change } \\
\text { lead3 }\end{array}$ & \begin{tabular}{r|}
-.087 \\
.215 \\
207
\end{tabular} & $\begin{array}{l}\text { USdollar__ } \\
\text { lead3 }\end{array}$ & $\begin{array}{r}-.068 \\
.329 \\
207\end{array}$ & $\begin{array}{l}\text { Bond_lea } \\
\text { d3 }\end{array}$ & $\begin{array}{l}.045 \\
.520 \\
207\end{array}$ & $\begin{array}{l}\text { Bank__ } \\
\text { credit_ } \\
\text { lead3 }\end{array}$ & $\begin{array}{r}-.065 \\
.354 \\
207\end{array}$ & $\begin{array}{l}\text { Property } \\
\text { lead3 }\end{array}$ & $\begin{array}{r}-.043 \\
.534 \\
207\end{array}$ & $\begin{array}{l}\text { Commodity } \\
\text { lead3 }\end{array}$ & $\begin{array}{r}-.017 \\
.803 \\
207\end{array}$ & $\begin{array}{l}\text { Gold_ } \\
\text { lead3 }\end{array}$ & $\begin{array}{l}.065 \\
.349 \\
207\end{array}$ \\
\hline $\begin{array}{l}\text { Pearson } \\
\text { Correlation } \\
\text { Sig. (2- } \\
\text { tailed) } \\
\mathrm{N}\end{array}$ & \begin{tabular}{|l} 
M1change_ \\
lead4
\end{tabular} & $\begin{array}{l}.033 \\
.641 \\
207\end{array}$ & $\begin{array}{l}\text { M2change } \\
\text { lead4 }\end{array}$ & \begin{tabular}{r|}
-.036 \\
.603 \\
207
\end{tabular} & $\begin{array}{l}\text { USdollar } \\
\text { lead4 }\end{array}$ & $\begin{array}{l}.015 \\
.834 \\
207\end{array}$ & $\begin{array}{l}\text { Bond_lea } \\
\text { d4 }\end{array}$ & $\begin{array}{l}.068 \\
.333 \\
207\end{array}$ & $\begin{array}{l}\text { Bank_ } \\
\text { credit_ } \\
\text { lead4 }\end{array}$ & $\begin{array}{r}-.048 \\
.495 \\
207\end{array}$ & $\begin{array}{l}\text { Property } \\
\text { lead4 }\end{array}$ & $\begin{array}{l}.029 \\
.683 \\
207\end{array}$ & $\begin{array}{l}\text { Commodity } \\
\text { lead4 }\end{array}$ & $\begin{array}{l}.009 \\
.901 \\
207\end{array}$ & $\begin{array}{l}\text { Gold__ } \\
\text { lead4 }\end{array}$ & $\begin{array}{l}.036 \\
.605 \\
207\end{array}$ \\
\hline $\begin{array}{l}\text { Pearson } \\
\text { Correlation } \\
\text { Sig. (2- } \\
\text { tailed) } \\
\mathrm{N}\end{array}$ & $\begin{array}{l}\text { M1change_ } \\
\text { lead5 }\end{array}$ & $\begin{array}{l}.064 \\
.363 \\
207\end{array}$ & $\begin{array}{l}\text { M2change } \\
\text { lead5 }\end{array}$ & \begin{tabular}{r|}
.073 \\
.297 \\
207
\end{tabular} & $\begin{array}{l}\text { USdollar_ } \\
\text { lead5 }\end{array}$ & $\begin{array}{r}-.168^{*} \\
.016 \\
207\end{array}$ & $\begin{array}{l}\text { Bond_lea } \\
\text { d5 }\end{array}$ & $\begin{array}{l}.153^{*} \\
.028 \\
207\end{array}$ & $\begin{array}{l}\text { Bank_ } \\
\text { credit_ } \\
\text { lead5 }\end{array}$ & $\begin{array}{l}.085 \\
.223 \\
207\end{array}$ & $\begin{array}{l}\text { Property } \\
\text { lead5 }\end{array}$ & $\begin{array}{r}-.017 \\
.809 \\
207\end{array}$ & $\begin{array}{l}\text { Commodity_ } \\
\text { lead5 }\end{array}$ & $\begin{array}{l}.237^{* *} \\
.001 \\
207\end{array}$ & $\begin{array}{l}\text { Gold__ } \\
\text { lead5 }\end{array}$ & $\begin{array}{l}.074 \\
.292 \\
207\end{array}$ \\
\hline $\begin{array}{l}\text { Pearson } \\
\text { Correlation } \\
\text { Sig. (2- } \\
\text { tailed) } \\
\mathrm{N}\end{array}$ & $\begin{array}{l}\text { M1change_ } \\
\text { lead6 }\end{array}$ & $\begin{array}{l}.133 \\
.057 \\
207\end{array}$ & $\begin{array}{l}\text { M2change_ } \\
\text { lead6 }\end{array}$ & \begin{tabular}{l|}
.006 \\
.926 \\
207
\end{tabular} & $\begin{array}{l}\text { USdollar_ } \\
\text { lead6 }\end{array}$ & $\begin{array}{l}.042 \\
.549 \\
207\end{array}$ & $\begin{array}{l}\text { Bond_lea } \\
\text { d6 }\end{array}$ & $\begin{array}{l}.027 \\
.699 \\
207\end{array}$ & $\begin{array}{l}\text { Bank_ } \\
\text { credit_ } \\
\text { lead6 }\end{array}$ & $\begin{array}{r}-.139^{*} \\
.047 \\
207\end{array}$ & $\begin{array}{l}\text { Property } \\
\text { lead6 }\end{array}$ & $\begin{array}{l}.104 \\
.137 \\
207\end{array}$ & $\begin{array}{l}\text { Commodity } \\
\text { lead6 }\end{array}$ & $\begin{array}{l}.043 \\
.539 \\
207\end{array}$ & $\begin{array}{l}\text { Gold_ } \\
\text { lead6 }\end{array}$ & $\begin{array}{l}-.015 \\
.832 \\
207\end{array}$ \\
\hline $\begin{array}{l}\text { Pearson } \\
\text { Correlation } \\
\text { Sig. (2- } \\
\text { tailed) } \\
\mathrm{N}\end{array}$ & \begin{tabular}{|l} 
M1chnage_ \\
lead7
\end{tabular} & $\begin{array}{r}-.190^{* *} \\
.006 \\
207\end{array}$ & $\begin{array}{l}\text { M2change_ } \\
\text { lead7 }\end{array}$ & \begin{tabular}{r|}
-.099 \\
.155 \\
207
\end{tabular} & $\begin{array}{l}\text { USdollar } \\
\text { lead7 }\end{array}$ & \begin{tabular}{r|}
-.063 \\
.371 \\
207
\end{tabular} & $\begin{array}{l}\text { Bond_lea } \\
\text { d7 }\end{array}$ & \begin{tabular}{r|}
-.123 \\
.078 \\
207
\end{tabular} & $\begin{array}{l}\text { Bank_ } \\
\text { credit_ } \\
\text { lead7 }\end{array}$ & $\begin{array}{r}-167^{*} \\
.016 \\
207\end{array}$ & $\begin{array}{l}\text { Property } \\
\text { lead7 }\end{array}$ & $\begin{array}{l}.126 \\
.071 \\
207\end{array}$ & $\begin{array}{l}\text { Commodity } \\
\text { lead7 }\end{array}$ & $\begin{array}{l}.075 \\
.285 \\
207\end{array}$ & $\begin{array}{l}\text { Gold__ } \\
\text { lead7 }\end{array}$ & $\begin{array}{l}.093 \\
.183 \\
207\end{array}$ \\
\hline $\begin{array}{l}\text { Pearson } \\
\text { Correlation } \\
\text { Sig. (2- } \\
\text { tailed) } \\
\mathrm{N}\end{array}$ & $\begin{array}{l}\text { M1change_ } \\
\text { lead8 }\end{array}$ & $\begin{array}{l}.024 \\
.726 \\
207\end{array}$ & $\begin{array}{l}\text { M2change_ } \\
\text { lead8 }\end{array}$ & \begin{tabular}{r|}
-.077 \\
.269 \\
207
\end{tabular} & $\begin{array}{l}\text { USdollar_ } \\
\text { lead8 }\end{array}$ & $\begin{array}{r}-.017 \\
.806 \\
207\end{array}$ & $\begin{array}{l}\text { Bond_lea } \\
\text { d8 }\end{array}$ & $\begin{array}{l}.044 \\
.528 \\
207\end{array}$ & $\begin{array}{l}\text { Bank__ } \\
\text { credit_ } \\
\text { lead8 }\end{array}$ & $\begin{array}{l}.001 \\
.987 \\
207\end{array}$ & $\begin{array}{l}\text { Property } \\
\text { lead8 }\end{array}$ & $\begin{array}{c}.038 \\
.582 \\
207\end{array}$ & $\begin{array}{l}\text { Commodity_ } \\
\text { lead8 }\end{array}$ & $\begin{array}{l}.145^{*} \\
.037 \\
207\end{array}$ & $\begin{array}{l}\text { Gold__ } \\
\text { lead8 }\end{array}$ & $\begin{array}{r}-.110 \\
.114 \\
207\end{array}$ \\
\hline $\begin{array}{l}\text { Pearson } \\
\text { Correlation } \\
\text { Sig. (2- } \\
\text { tailed) } \\
\mathrm{N}\end{array}$ & $\begin{array}{l}\text { M1change_ } \\
\text { lead9 }\end{array}$ & $\begin{array}{r}.040 \\
.568 \\
207\end{array}$ & $\begin{array}{l}\text { M2chnage_ } \\
\text { lead9 }\end{array}$ & \begin{tabular}{r|}
-.076 \\
.275 \\
207
\end{tabular} & $\begin{array}{l}\text { USdollar_ } \\
\text { lead9 }\end{array}$ & $\begin{array}{l}.089 \\
.203 \\
207\end{array}$ & $\begin{array}{l}\text { Bond_lea } \\
\text { d9 }\end{array}$ & $\begin{array}{l}.052 \\
.455 \\
207\end{array}$ & $\begin{array}{l}\text { Bank_ } \\
\text { credit_ } \\
\text { lead9 }\end{array}$ & $\begin{array}{l}.071 \\
.313 \\
207\end{array}$ & $\begin{array}{l}\text { Property } \\
\text { lead9 }\end{array}$ & $\begin{array}{l}.093 \\
.185 \\
207\end{array}$ & $\begin{array}{l}\text { Commodity } \\
\text { lead9 }\end{array}$ & $\begin{array}{r}-.105 \\
.132 \\
207\end{array}$ & $\begin{array}{l}\text { Gold_ } \\
\text { lead9 }\end{array}$ & $\begin{array}{r}-.015 \\
.834 \\
207\end{array}$ \\
\hline $\begin{array}{l}\text { Pearson } \\
\text { Correlation } \\
\text { Sig. (2- } \\
\text { tailed) } \\
\mathrm{N}\end{array}$ & $\begin{array}{l}\text { M1change_ } \\
\text { lead10 }\end{array}$ & $\begin{array}{l}-.027 \\
.700 \\
207\end{array}$ & $\begin{array}{l}\text { M2change_ } \\
\text { lead10 }\end{array}$ & \begin{tabular}{r|}
.070 \\
.314 \\
207
\end{tabular} & $\begin{array}{l}\text { USdollar_ } \\
\text { lead10 }\end{array}$ & $\begin{array}{l}.113 \\
.104 \\
207\end{array}$ & $\begin{array}{l}\text { Bond_lea } \\
\text { d10 }\end{array}$ & $\begin{array}{l}.033 \\
.639 \\
207\end{array}$ & $\begin{array}{l}\text { Bank_ } \\
\text { credit_- } \\
\text { lead10 }\end{array}$ & $\begin{array}{r}-.060 \\
.389 \\
207\end{array}$ & $\begin{array}{l}\text { Property } \\
\text { lead } 10\end{array}$ & $\begin{array}{r}-.150^{2} \\
.031 \\
207\end{array}$ & $\begin{array}{l}\text { Commodity } \\
\text { lead10 }\end{array}$ & $\begin{array}{l}.077 \\
.268 \\
207\end{array}$ & $\begin{array}{l}\text { Gold_ } \\
\text { lead10 }\end{array}$ & $\begin{array}{r}-.101 \\
.150 \\
207\end{array}$ \\
\hline $\begin{array}{l}\text { Pearson } \\
\text { Correlation } \\
\text { Sig. (2- } \\
\text { tailed) } \\
\mathrm{N}\end{array}$ & \begin{tabular}{|l} 
M1change_ \\
lead11
\end{tabular} & $\begin{array}{l}.128 \\
.065 \\
207\end{array}$ & $\begin{array}{l}\text { M2change } \\
\text { lead11 }\end{array}$ & \begin{tabular}{l|}
.046 \\
.508 \\
207
\end{tabular} & $\begin{array}{l}\text { USdollar_ } \\
\text { lead11 }\end{array}$ & $\begin{array}{l}. .131 \\
.060 \\
207\end{array}$ & $\begin{array}{l}\text { Bond_lea } \\
\text { d11 }\end{array}$ & $\begin{array}{l}.013 \\
.851 \\
207\end{array}$ & $\begin{array}{l}\text { Bank_ } \\
\text { credit__ } \\
\text { lead11 }\end{array}$ & $\begin{array}{r}.145^{*} \\
.038 \\
207\end{array}$ & $\begin{array}{l}\text { Property } \\
\text { lead11 }\end{array}$ & $\begin{array}{l}.096 \\
.168 \\
207\end{array}$ & $\begin{array}{l}\text { Commodity } \\
\text { lead11 }\end{array}$ & $\begin{array}{r}.188^{* *} \\
.007 \\
207\end{array}$ & $\begin{array}{l}\text { Gold_ } \\
\text { lead11 }\end{array}$ & $\begin{array}{l}.066 \\
.345 \\
207\end{array}$ \\
\hline $\begin{array}{l}\text { Pearson } \\
\text { Correlation } \\
\text { Sig. (2- } \\
\text { tailed) } \\
\text { N }\end{array}$ & $\begin{array}{l}\text { M1change_ } \\
\text { lead12 }\end{array}$ & $\begin{array}{l}.006 \\
.931 \\
207\end{array}$ & $\begin{array}{l}\text { M2change_ } \\
\text { lead12 }\end{array}$ & \begin{tabular}{r|}
-.021 \\
.765 \\
207
\end{tabular} & $\begin{array}{l}\text { USdollar_ } \\
\text { lead12 }\end{array}$ & $\begin{array}{c}.100 \\
.151 \\
207\end{array}$ & $\begin{array}{l}\text { Bond_lea } \\
\text { d12 }\end{array}$ & $\begin{array}{l}. .131 \\
.059 \\
207\end{array}$ & $\begin{array}{l}\text { Bank_ } \\
\text { credit_ } \\
\text { lead12 }\end{array}$ & $\begin{array}{l}.083 \\
.236 \\
207\end{array}$ & $\begin{array}{l}\text { Property } \\
\text { lead12 }\end{array}$ & $\begin{array}{l}.081 \\
.248 \\
207\end{array}$ & $\begin{array}{l}\text { Commodity } \\
\text { lead12 }\end{array}$ & $\begin{array}{l}.111 \\
.112 \\
207\end{array}$ & $\begin{array}{l}\text { Gold_ } \\
\text { lead12 }\end{array}$ & $\begin{array}{l}.072 \\
.304 \\
207\end{array}$ \\
\hline $\begin{array}{l}\text { Pearson } \\
\text { Correlation } \\
\text { Sig. (2- } \\
\text { tailed) } \\
\mathrm{N}\end{array}$ & $\begin{array}{l}\text { M1change_ } \\
\text { lead13 }\end{array}$ & $\begin{array}{l}.031 \\
.653 \\
207\end{array}$ & $\begin{array}{l}\text { M2change_ } \\
\text { lead13 }\end{array}$ & \begin{tabular}{r|}
.011 \\
.876 \\
207
\end{tabular} & $\begin{array}{l}\text { USdollar_ } \\
\text { lead13 }\end{array}$ & $\begin{array}{c}-.120 \\
.085 \\
207\end{array}$ & $\begin{array}{l}\text { Bond_lea } \\
\text { d13 }\end{array}$ & $\begin{array}{l}.112 \\
.108 \\
207\end{array}$ & $\begin{array}{l}\text { Bank_ } \\
\text { credit_ }_{-} \\
\text {lead13 }\end{array}$ & $\begin{array}{l}-.037 \\
.600 \\
207\end{array}$ & $\begin{array}{l}\text { Property } \\
\text { lead13 }\end{array}$ & $\begin{array}{c}-.041 \\
.561 \\
207\end{array}$ & $\begin{array}{l}\text { Commodity_ } \\
\text { lead13 }\end{array}$ & $\begin{array}{l}.054 \\
.443 \\
207\end{array}$ & $\begin{array}{l}\text { Gold_ } \\
\text { lead13 }\end{array}$ & $\begin{array}{l}.085 \\
.221 \\
207\end{array}$ \\
\hline $\begin{array}{l}\text { Pearson } \\
\text { Correlation } \\
\text { Sig. (2- } \\
\text { tailed) } \\
\text { N }\end{array}$ & $\begin{array}{l}\text { M1change_ } \\
\text { lag1 }\end{array}$ & $\begin{array}{l}.020 \\
.780 \\
207\end{array}$ & $\begin{array}{l}\text { M2change_ } \\
\text { lag1 }\end{array}$ & \begin{tabular}{r|}
$-.146^{*}$ \\
.035 \\
207
\end{tabular} & $\begin{array}{l}\text { USdollar_ } \\
\text { lag1 }\end{array}$ & \begin{tabular}{r|}
$-.159^{2}$ \\
.022 \\
207
\end{tabular} & $\begin{array}{l}\text { Bond_lag } \\
1\end{array}$ & $\begin{array}{l}.004 \\
.953 \\
207\end{array}$ & $\begin{array}{l}\text { Bank__ } \\
\text { credit_ } \\
\text { lag1 }\end{array}$ & $\begin{array}{l}.031 \\
.657 \\
207\end{array}$ & $\begin{array}{l}\text { Property } \\
\text { lag1 }\end{array}$ & $\begin{array}{r}.180^{3 *} \\
.009 \\
207\end{array}$ & $\begin{array}{l}\text { Commodity_ } \\
\text { lag1 }\end{array}$ & $\begin{array}{l}.128 \\
.065 \\
207\end{array}$ & $\begin{array}{l}\text { Gold_1 } \\
\text { ag1 }\end{array}$ & $\begin{array}{r}-.035 \\
.615 \\
207\end{array}$ \\
\hline $\begin{array}{l}\text { Pearson } \\
\text { Correlation } \\
\text { Sig. (2- } \\
\text { tailed) } \\
\mathrm{N}\end{array}$ & $\begin{array}{l}\text { M1change } \\
\text { lag2 }\end{array}$ & $\begin{array}{c}-.043 \\
.540 \\
207\end{array}$ & $\begin{array}{l}\text { M2change } \\
\text { lag2 }\end{array}$ & $\begin{array}{r}-.221^{* *} \\
.001 \\
207\end{array}$ & $\begin{array}{l}\text { USdollar_ } \\
\text { lag2 }\end{array}$ & $\begin{array}{l}.024 \\
.730 \\
207\end{array}$ & $\begin{array}{l}\text { Bond_lag } \\
2\end{array}$ & \begin{tabular}{r|}
$.139^{*}$ \\
.045 \\
207
\end{tabular} & $\begin{array}{l}\text { Bank_- } \\
\text { credit_- } \\
\text { lag2 }\end{array}$ & $\begin{array}{r}-.028 \\
.690 \\
207\end{array}$ & $\begin{array}{l}\text { Property } \\
\text { lag2 }\end{array}$ & $\begin{array}{l}.050 \\
.476 \\
207\end{array}$ & $\begin{array}{l}\text { Commodity } \\
\text { lag2 }\end{array}$ & $\begin{array}{l}.025 \\
.716 \\
207\end{array}$ & $\begin{array}{l}\text { Gold_1 } \\
\text { ag2 }\end{array}$ & $\begin{array}{l}.027 \\
.701 \\
207\end{array}$ \\
\hline \begin{tabular}{|l} 
Pearson \\
Correlation
\end{tabular} & $\begin{array}{l}\text { M1change } \\
\text { lag3 }\end{array}$ & .009 & $\begin{array}{l}\text { M2change_ } \\
\text { lag3 }\end{array}$ & $-.138^{*}$ & $\begin{array}{l}\text { USdollar } \\
\text { lag3 }\end{array}$ & .004 & $\begin{array}{l}\text { Bond_lag } \\
3\end{array}$ & .095 & $\begin{array}{l}\text { Bank__ } \\
\text { credit__ }\end{array}$ & .024 & $\begin{array}{l}\text { Property } \\
\text { lag3 }\end{array}$ & -.055 & $\begin{array}{l}\text { Commodity } \\
\text { lag3 }\end{array}$ & .130 & $\begin{array}{l}\text { Gold_1 } \\
\text { ag3 }\end{array}$ & -.012 \\
\hline
\end{tabular}




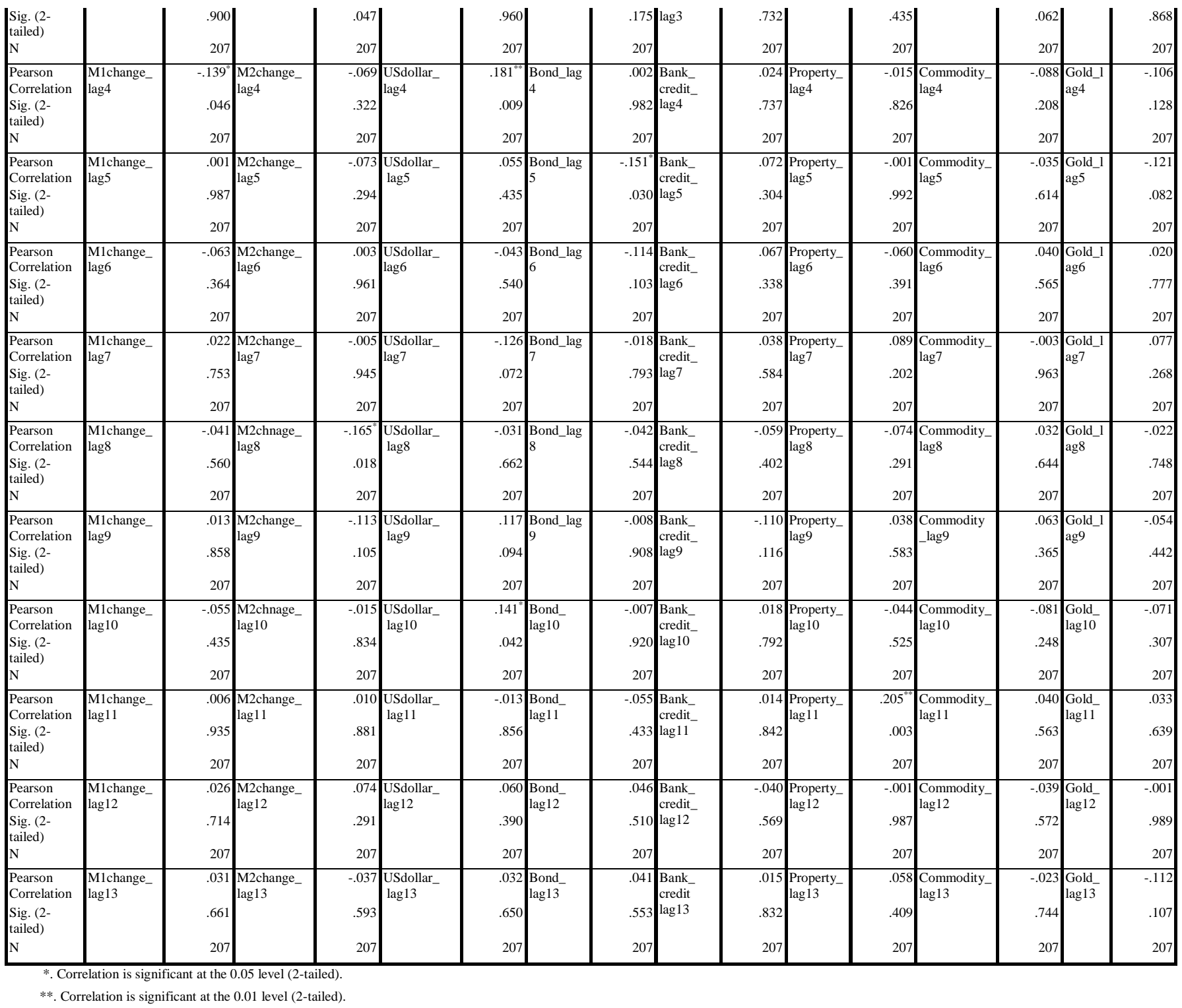

Next step is to test the stationarity or non-stationarity of the variables' time series in Panel A and Panel B. The ADF tests are conducted. The time series of all lead-lag variables in two panels are stationary.

Use the above variables as the inputs to run a regression for each panel. Table 6 shows the result of Panel A. From the heading Collinearity Statistics in the Coefficients box, the highest Tolerance value and the lowest VIF are 0.636 and 1.572. Multicollinearity does not exist in the regression. In the Model Summary box, the R Square is 0.507 and Adjusted R Square is 0.49 . 50.7\% of the variances in the SP500_return can be predicted by the variables. In the ANOVA box, the value of Sig is 0.000 , which is less than 0.05 .

This is sufficient to conclude that there is a significant relationship between the variables and SP500_return. Assessing the significance of individual parameters, all of them are significant (Sig <0.05) except the Bond_lead 4 (Sig=0.415). Therefore, the regression equation can be written as:

$S P 500 \_$return $=0.004-0.503 M 1$ change_lead $12-1.448 M 2$ change_lead 1

-0.514 USdollar_change-1.376Bank_credit_lead $4+0.206$ Property_lead 1

+0.212 Commodity_return +0.237 Gold_lag 3

Table 7 shows the result of Panel B. The highest Tolerance value and the lowest VIF are 0.621 and 1.609. Multicollinearity does not exist in the regression. The R Square is 0.498 and Adjusted R Square is $0.478 .49 .8 \%$ of the variances in the SP500_return can be predicted by the variables. In the ANOVA box, the value of Sig is 0.000, which is 
less than 0.05. This is sufficient to conclude that there is a significant relationship between the variables and SP500_return. Assessing the significance of individual parameters, M2change_lag 2 (Sig=0.168) and Bank_credit_lead 7 (Sig=0.07) are insignificant while the others are significant $(\mathrm{Sig}<0.05)$. The regression equation can be written as

$$
\begin{aligned}
& \text { SP500_return }=0.005-0.504 M 1 \text { change_lead } 7-0.544 U \text { Sdollar_change } \\
& -0.838 \text { Bond_lead } 1+0.178 \text { Property_lead } 1+0.27 \text { Commodity_return } \\
& +0.137 \text { Gold_lead } 2
\end{aligned}
$$

The two results show that the explanations of the models in Panel A and Panel B are better than the original one. From the result of Table 8, the Chow test also support that the structure of the two panels is different. The relationship between SP500_return and independent variables changes according to different investment environments.

Table 6 Multiple Regression Result of Panel A

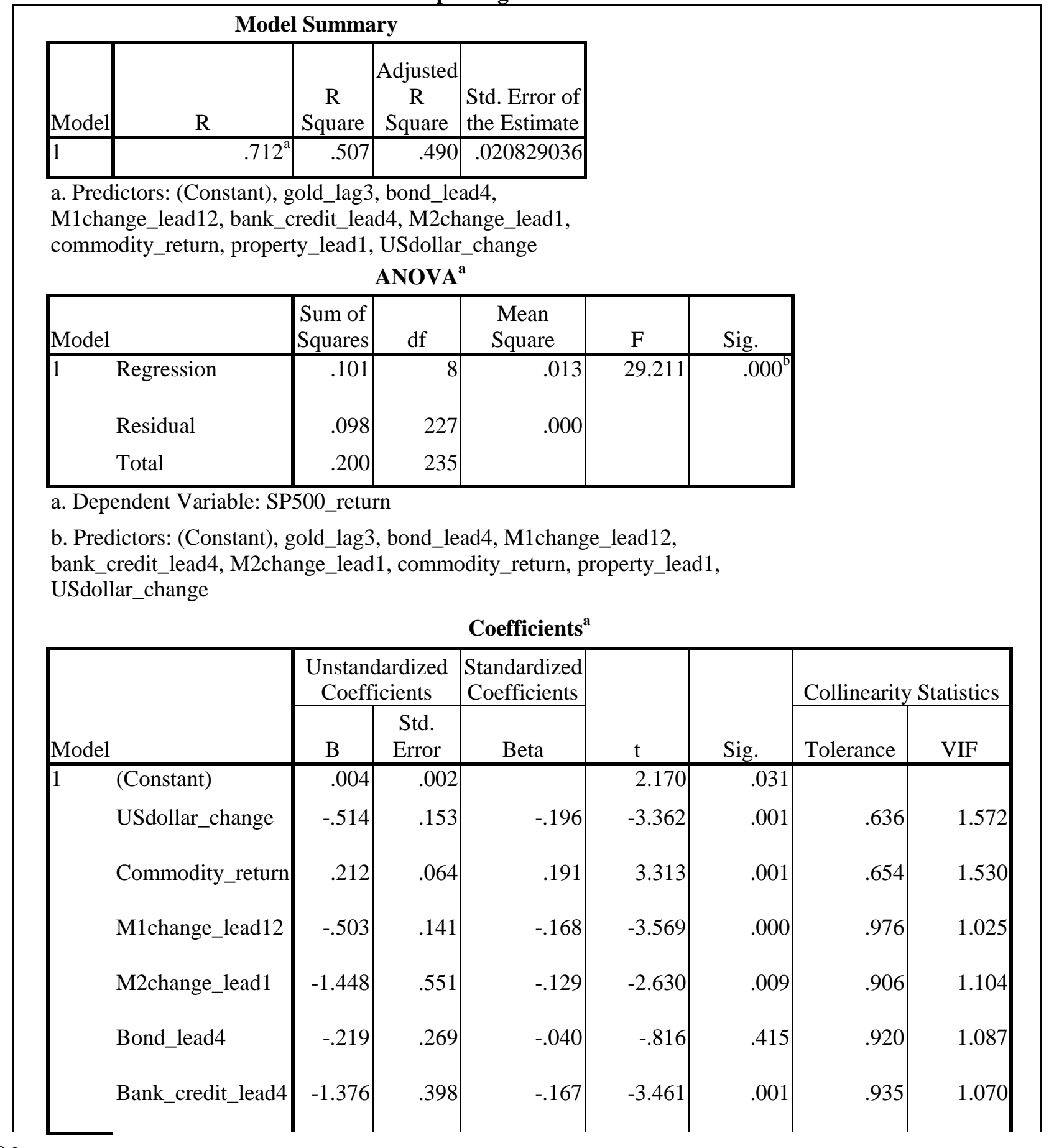




\begin{tabular}{|c|r|r|r|r|r|r|r|} 
Property_lead1 & .206 & .033 & .307 & 6.220 & .000 & .890 & 1.124 \\
Gold_lag3 & .237 & .045 & .255 & 5.333 & .000 & .949 & 1.054 \\
\hline a. Dependent Variable: SP500_return
\end{tabular}

Table 7 Multiple Regression Result of Panel B

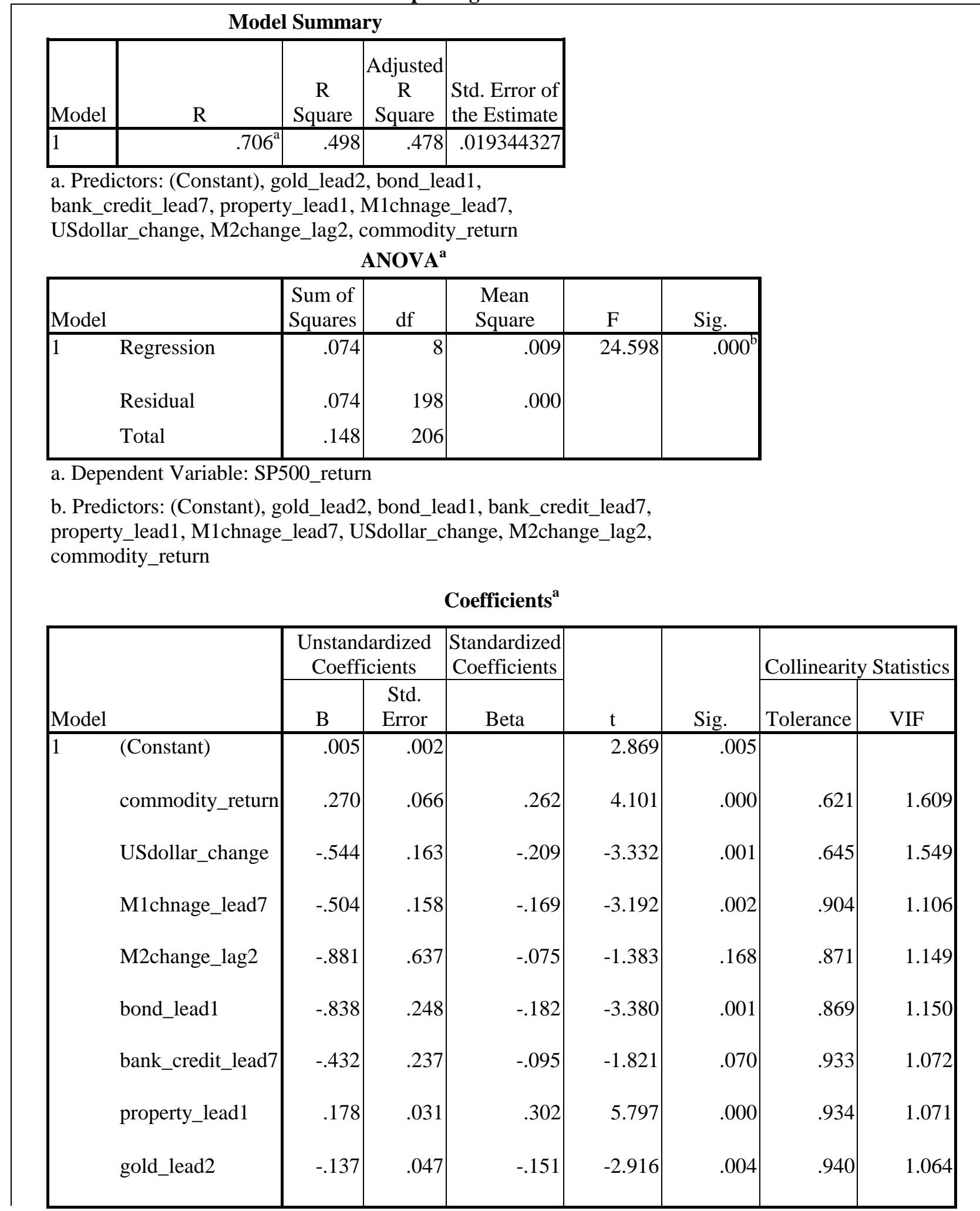


a. Dependent Variable: SP500_return

Table 8. Chow Test result for Panel A and Panel B

Regression stability test (Chow test)

\begin{tabular}{ccccc}
\hline Score & C.V. & P-Value & Stable? & $\mathbf{5 . 0 \%}$ \\
\hline 0.000 & 1.962 & $0.00 \%$ & FALSE &
\end{tabular}

\section{Conclusion}

In this research, eight variables are applied to evaluate the effect of capital flow on the US stock market return. In the first part, Pearson Correlations and regression analysis are conducted between the dependent variable and independent variables. In the second part, the Pearson Correlations and regression analysis are repeated to explore whether there are lead-lag relationships between them. The findings show that six out of eight variables have the lead-lag relationship with the stock market return. However, one constant coefficient correlation between the dependent variable and independent variables for the whole period is misleading. Therefore, I divide the period into two panels reflecting different investment environments. Panel A is when the growth rate of M1 greater than the growth rate of M2 representing that the market has sufficient funds while Panel B is when the growth rate of M2 greater than the growth rate of M1representing that the market has lesser funds. From the results, most of the eight variables in the two panels have a more slightly correlated relationship with the stock market return. More important is that the highest coefficient correlation of Gold_return with SP_500 return is positive in Panel A and is negative in Panel B. This result is more able to prove that the correlations between the dependent variable and independent variables will change under different investment environments. This also explains why the prediction ability of the variables in the two panels is stronger than the original data set.

\section{Bibliography}

Abdullah, D.A. and Hayworth, S.C. (1993) Macroeconomics of Stock Price Fluctuation. Quarterly Journal of Business and Economics, 32(1), 50-67

Alshogeathri, M.A.M., (2011)Macroeconomic determinants of the stock market movements: Empirical evidence from the Saudi stock market, Kansas State University.

Baur, D. and Lucey, B. M. (2006) Flight-to-quality or Contagion? An Empirical Analysis of Stock-Bond Correlations Working Research, Trinity College, Dublin.

Baur, D. and Mcdermott, T.K., 2010. Is gold a safe haven? International evidence. Journal of Banking and Finance, 34(8), pp. 1886-1898

Beracha, E., (2007)Essays on capital markets and real estate finance, University of Kansas.

Capie, F., Mills, T.C. and Wood, G., 2005.Gold as a hedge against the dollar. Journal of international financial markets, institutions and money, 15(4), pp. 343-352.

Cohen, J.W. (1988). Statistical power analysis for the behavioral sciences $\left(2^{\text {nd }}\right.$ edn). Hillsdale, NJ: Lawrence Erlbaum Associates.

Connolly, R.A., Stivers, C.T. and Sun, L. (2005) Stock Market Uncertainly and the Stock-Bond Return Relation Journal of Financial and Quantitative analysis, 40, 161-194

Coudert, V. and Raymond, H., (2012) Gold, is it a safe haven during recessions and stock market crashes? Revue économique, 63(5), pp. 993-1011.

Culver, M.P., (2010)The global credit crisis of 2007--2009: An examination of some of the causes, chronology, and unconventional monetary tools employed, University of Denver.

Darrat, A.F. and Dickens, R.N. (1999( On the Interrelationships among Real, Monetary, and Financial Variables Applied Financial Economics 9(3), 289-293

Dhakai, D., Kandil, M. and Sharma, S.C. (1993) Causality between the Money Supply and Share Prices: A VAR Investigation. Quarterly Journal of Business and Economics, 32(3), 52-74

Downing C., Underwood, S. and Xing, Y. (2006) Is Liquidity Risk Priced in the Corporate Bond Market? Working Research, Rice University.

Gerig, A.N. (2007)A theory for market impact: How order flow affects stock price, University of Illinois at UrbanaChampaign.

Gulko, L. (2002) Decoupling The Journal of Portfolio Management, 28, 59-66.

Hartmann, P., Straetmann, S. and Devries, C.G. "Asset Market Linkages in Crisis Periods."Working Research, ECB \#71. (2001). 
Hashemzadeh, N. and Taylor, P. (1988) Stock Prices, Money Supply, and Interest Rate: the Question of Causality. Applied Economics, 20, 1603-1611

Hillier, D., Draper, P. and Faff, R., 2006. Do precious metals shine? An investment perspective. Financial analysts journal, 62(2), pp. 98-111.

Hondroyiannis, G. and Papapetrou, E. (2001) Macroeconomic Influences on the Stock Market Journal of Economics and Finance, 25(1), 33-49

Ibrahim, M.H. (1999) Macroeconomic Variables and Stock Prices in Malaysia: An Empirical Analysis Asian Economic Journal, 13(2), 219-231.

Ibrahim, M.H. (2006) Stock Price and Bank Loan Dynamics in a Developing Country: The Case of Malaysia Journal of Applied Economics, IX(1), 71-89.

Kim, S. B. and Moreno, R. (1994) Stock Prices and Bank Lending Behavior in Japan Review, Federal Reserve Bank of San Francisco, CA, 31-42.

LI, J., (2007)Three essays on modeling stock returns: Empirical analysis of the residual distribution, risk-return relation, and stock-bond dynamic correlation, Drexel University.

LI, Z., 2013. European Stock Market Contagion during Sovereign Debt Crisis and the Effects of Macroeconomic Announcements on the Correlations of Gold, Dollar and Stock Returns, University of New Orleans.

Lombardi, M. and Ravazzolo, F. (2013) On the correlation between commodity and equity returns: implications for portfolio allocation Bank for International Settlements, BIS Working Research, 420.

Malliaris, A.G. andUrrutia, J.L.(1991) An Empirical Investigation among Real, Monetary and

Financial Variables Economics Letters, Elsevier, 37(2), 151-158

Milunovich, G., Passe-De Silva, A. and Heaton, C., 2011. International commodity prices and the Australian stock market. Economic record, 87(276), pp. 37-44.

Oh, N.Y. \&Parwada, J.T.(2007) "Relations between mutual fund flows and stock market returns in Korea", Journal of international financial markets, institutions and money, vol. 17, no. 2, pp. 140-151.

Patra, T. and Poshakwale, S. (2006) Economic Variables and Stock Market Returns: Evidence from the Athens Stock Exchange Applied Financial Economics, 16, 993-1005

Ratanapakorn, O. and Sharma, S. (2007) Dynamic Analysis between the US Stock Returns and the Macroeconomic Variables, Applied Financial Economics, 17(5), 369-377

Stevenson, A.J., 2004. Price relationships between resource based stock prices and commodity prices, University of Manitoba (Canada).

Sutton, G.D. (2002) Explaining Changes in House Prices Bis Quarterly Review(September): 46-55.

Tabachnick, B. G., and Fidell, L.S. (2001) Using multivariate statistics $\left(4^{\text {th }}\right.$ edn $)$ New York: HarperCollins.

Thornton, J. (1993) Money, Output and Stock Prices in the UK: Evidence on Some (Non) Relationships, Applied Financial Economics, 3, 335-338.

Tas, D., (2008)Essays on exchange rate risk, asset returns and trade flows in East Asian emerging market economies, Southern Illinois University at Carbondale

Wenshwo, F. (2002) The Effects of Currency Depreciation on Stock Returns: Evidence from Five East Asian Economics Applied Economics Letters, 9(3), 195-199.

YIN, H., (2007) The effect of changes in Federal funds rate target on bank stock returns in the U.S.A, The George Washington University. 